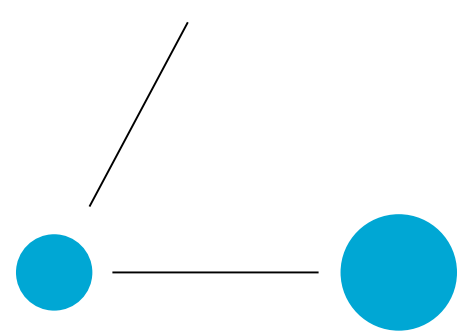

A N N A L E S HENRI LEBESGUE

SHU NAKAMURA

\title{
ESSENTIAL SELF-ADJOINTNESS OF REAL PRINCIPAL TYPE OPERATORS
}

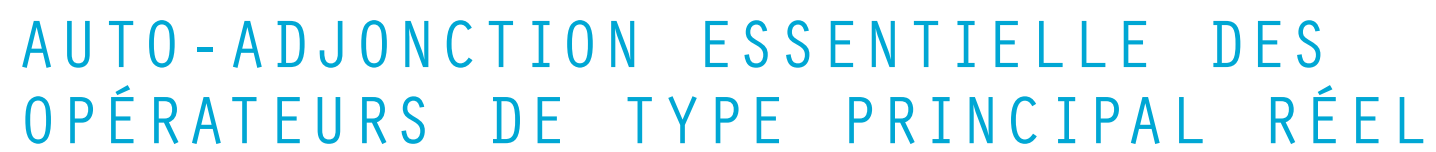

Abstract. - We study the essential self-adjointness for real principal type differential operators. Unlike the elliptic case, we need geometric conditions even for operators on the Euclidean space with asymptotically constant coefficients, and we prove the essential selfadjointness under the null non-trapping condition.

RÉsumÉ. - Nous étudions le caractère essentiellement auto-adjoint pour des opérateurs de type principal réel. Contrairement au cas elliptique, nous avons besoin de conditions géométriques même pour des opérateurs sur l'espace euclidien avec coefficients asymptotiquement constants, et nous démontrons le caractère essentiellement auto-adjoint sous la condition de non-capture à énergie zéro.

Keywords: Principal type operators, essential self-adjointness, non-trapping conditions. 2020 Mathematics Subject Classification: 58J50, 35P25, 47B25.

DOI: https://doi.org/10.5802/ahl.96

(*) SN is partially supported by JSPS grant Kiban-B 15H03622. KT is supported by JSPS Research Fellowship for Young Scientists, KAKENHI Grant Number 17J04478 and the program FMSP at the Graduate School of Mathematics Sciences, the University of Tokyo. 


\section{Introduction}

In this paper, we consider formally self-adjoint real principal type operator $P=$ $\mathrm{Op}(p)$ on the Euclidean space $\mathbb{R}^{n}$ with $n \geqslant 1$, where $\mathrm{Op}(\cdot)$ denotes the Weyl quantization. A typical example is the Klein-Gordon operator with variable coefficients (see Remark 1.4), and the propagation of singularities plays an essential role in the proof of the essential self-adjointness.

We suppose the symbol $p(x, \xi)$ is real principal type with asymptotically constant coefficients in the following sense:

Assumption 1.1. - Let $m \geqslant 2, p, p_{m} \in C^{\infty}\left(\mathbb{R}^{2 n}\right)$ and $p_{0} \in C^{\infty}\left(\mathbb{R}^{n}\right)$ be realvalued functions of the form

$$
p(x, \xi)=\sum_{|\alpha| \leqslant m} a_{\alpha}(x) \xi^{\alpha}, p_{m}(x, \xi)=\sum_{|\alpha|=m} a_{\alpha}(x) \xi^{\alpha}, p_{0}(\xi)=\sum_{|\alpha|=m} b_{\alpha} \xi^{\alpha}
$$

where $b_{\alpha} \in \mathbb{R}$ and $a_{\alpha} \in C^{\infty}\left(\mathbb{R}^{n}\right)$ such that for any multi-index $\beta \in \mathbb{Z}_{+}^{n}$,

$$
\left|\partial_{x}^{\beta}\left(a_{\alpha}(x)-b_{\alpha}\right)\right| \leqslant C_{\beta}\langle x\rangle^{-\mu-|\beta|}, \quad x \in \mathbb{R}^{n}
$$

with some $\mu>0$, where we set $b_{\alpha}=0$ for $|\alpha| \leqslant m-1$. Moreover, there exists $C>0$ such that

$$
C^{-1}|\xi|^{m-1} \leqslant\left|\partial_{\xi} p_{m}(x, \xi)\right| \leqslant C|\xi|^{m-1}
$$

for $(x, \xi) \in \mathbb{R}^{2 n}$. In particular, this implies

$$
C^{-1}|\xi|^{m-1} \leqslant\left|\partial_{\xi} p_{0}(\xi)\right| \leqslant C|\xi|^{m-1}
$$

Let $(y(t), \eta(t))=\left(y\left(t, x_{0}, \xi_{0}\right), \eta\left(t, x_{0}, \xi_{0}\right)\right) \in C^{1}\left(\mathbb{R} \times \mathbb{R}^{2 n} ; \mathbb{R}^{2 n}\right)$ be the solution to the Hamilton equation:

$$
\frac{d}{d t} y(t)=\frac{\partial p_{m}}{\partial \xi}(y(t), \eta(t)), \quad \frac{d}{d t} \eta(t)=-\frac{\partial p_{m}}{\partial x}(y(t), \eta(t)), \quad t \in \mathbb{R},
$$

with the initial condition: $(y(0), \eta(0))=\left(x_{0}, \xi_{0}\right) \in \mathbb{R}^{2 n}$. We suppose the following null non-trapping condition:

Assumption 1.2. - For any $\left(x_{0}, \xi_{0}\right) \in p_{m}^{-1}(0)$ with $\xi_{0} \neq 0,\left|y\left(t, x_{0}, \xi_{0}\right)\right| \rightarrow \infty$ as $|t| \rightarrow \infty$.

Our main theorem is the following:

Theorem 1.3. - Suppose Assumption 1.1 and 1.2. Then $P=\mathrm{Op}(p)$ is essentially self-adjoint on $C_{c}^{\infty}\left(\mathbb{R}^{n}\right)$.

Remark 1.4. - (Klein-Gordon operators on asymptotically Minkowski spaces) Let $g_{0}$ be the Minkowski metric on $\mathbb{R}^{n}: g_{0}=d x_{1}^{2}-d x_{2}^{2}-\cdots-d x_{n}^{2}$ and $g^{-1}=\left(g_{0}^{i j}\right)_{i, j=1}^{n}$ be its dual metric. A Lorentzian metric $g$ on $\mathbb{R}^{n}$ is called asymptotically Minkowski if $g^{-1}(x)=\left(g^{i j}(x)\right)_{i, j=1}^{n}$ satisfies, for any $\alpha \in \mathbb{Z}_{+}^{n}$ there is $C_{\alpha}>0$ such that

$$
\left|\partial_{x}^{\alpha}\left(g^{i j}(x)-g_{0}^{i j}\right)\right| \leqslant C_{\alpha}\langle x\rangle^{-\mu-|\alpha|}, \quad x \in \mathbb{R}^{n},
$$

with some $\mu>0$. Suppose $V(x), A_{j}(x) \in C^{\infty}\left(\mathbb{R}^{n} ; \mathbb{R}\right), j=1, \ldots, n$, such that

$$
\left|\partial_{x}^{\alpha} V(x)\right| \leqslant C_{\alpha}\langle x\rangle^{-\mu-|\alpha|}, \quad\left|\partial_{x}^{\alpha} A_{j}(x)\right| \leqslant C_{\alpha}\langle x\rangle^{-\mu-|\alpha|}, \quad x \in \mathbb{R}^{n},
$$


for any $\alpha \in \mathbb{Z}_{+}^{n}$. Then the symbol

$$
p(x, \xi)=\sum_{j, k=1}^{n} g^{j k}(x)\left(\xi_{j}-A_{j}(x)\right)\left(\xi_{k}-A_{k}(x)\right)+V(x)
$$

satisfies Assumption 1.1. The essential self-adjointness for this model is studied by Vasy [Vas20].

Remark 1.5. - In this paper, we only deal with operators with order greater than 1. The essential self-adjointness of first order operators on $C_{c}^{\infty}\left(\mathbb{R}^{n}\right)$ can be proved by Nelson's commutator theorem with its conjugate operator $N=-\Delta+|x|^{2}+1([\mathrm{RS} 80$, Theorem X.36]). We also note that if $P$ commutes with the complex conjugation: $P \bar{u}=\overline{P u}$, then, it is enough to assume the forward null non-trapping condition only instead of null non-trapping condition (cf. [RS80, Theorem X.3]).

Remark 1.6. - It is an open question whether Assumption 1.2 is necessary for essential self-adjointness of $P$.

The study of essential self-adjointness has a long history but mostly on operators of elliptic type (see [RS80, Chapter X and reference therein]). For the construction of solutions to evolution equation with real principal type operators, we refer the classical paper [DH72] by Duistermaat and Hörmander, and the textbook by Hörmander [Hör85]. Chihara [Chi02] studies the well-posedness and the local smoothing effects of the Schrödinger-type equations: $\partial_{t} u(t, x)=-i P u(t, x)$ under the globally non-trapping condition. The well-posedness implies essential self-adjointness of $P$ if the operator $P$ is symmetric. We assume the non-trapping condition only for null trajectories, since the microlocally elliptic region should not be relevant.

Recently, the scattering theory for Klein-Gordon operators on Lorentzian manifolds has been studied by several authors (see, e.g., [BVW15, GRHV16, Vas20] and references therein). We also mention related work on Strichartz estimates for Lorentzian manifolds ([GT12, MT15, Tai20]), nonlinear Schrödinger-type equations with Minkowski metric ([GS93, Sal07, Wan13]), and quantum field theory on Minkowski spaces ([GW19, VW18]). In order to study spectral properties of such equations or operators, self-adjointness is fundamental. We note a sufficient condition for the essential self-adjointness is discussed in Taira [Tai20]. The essential self-adjointness for Klein-Gordon operators on scattering Lorentzian manifolds is proved by Vasy [Vas20] under the same null non-trapping condition. We had independently found a proof of the essential self-adjointness using different method for compactly supported perturbations (we discuss the basic idea in Appendix C). Inspired by discussions with Vasy during 2017, we generalized the model to include long-range perturbations, and also to higher order real principal type operators. Our proof is considerably different from [Vas20], relatively self-contained, and hopefully simpler even though our result is more general than [Vas20] for the $\mathbb{R}^{n}$ case.

This paper is constructed as follows: in Section 2, we prepare several notations and basic lemmas. Our main result is proved in Section 3. In Subsection 3.1 we show that $(P-i) u=0$ implies $u$ is smooth. The basic idea of the proof is analogous to Nakamura [Nak05] on microlocal smoothing estimates, and relies on the construction of time-global escaping functions (see also Ito, Nakamura [IN09] for related results for 
scattering manifolds). The technical detail is given in Appendix B. In Subsection 3.2, we show the local smoothness implies an weighted Sobolev estimate, which is sufficient for the proof of the essential self-adjointness. The idea is analogous to the radial point estimates of Melrose [Mel94], and also related to the positive commutators method of Mourre. Here we construct weight functions explicitly to show necessary operator inequalities. The proof relies on the standard pseudodifferential operator calculus. In Appendix A, we prove non-trapping estimates for the classical trajectories generated by $p_{m}(x, \xi)$, which are necessary in Appendix B. The main lemma (Lemma A.2) is a generalization of a result by Kenig, Ponce, Rolvung and Vega [KPRV05], though the proof is significantly simplified. In Appendix C, we give a simplified proof of the essential self-adjointness for the compactly supported perturbation case. In this case the relatively involved argument of Subsection 3.2 is not necessarily.

\section{Acknowledgments}

We are grateful to Andraś Vasy for stimulating discussions during RIMS meeting at Kyoto in 2017. We also would like to thank referees for suggesting improvement of this paper.

\section{Preliminary}

We set $\langle x\rangle=\left(1+|x|^{2}\right)^{1 / 2}$ and $D_{x}=-i \partial / \partial x$. We denote the weighted Sobolev spaces by

$$
H^{s, \ell}=H^{s, \ell}\left(\mathbb{R}^{n}\right)=\langle x\rangle^{-\ell}\left\langle D_{x}\right\rangle^{-s}\left[L^{2}\left(\mathbb{R}^{n}\right)\right],
$$

for $s, \ell \in \mathbb{R}$, and their norms are given by

$$
\|\varphi\|_{H^{s, \ell}}=\left\|\left\langle D_{x}\right\rangle^{s}\langle x\rangle^{\ell} \varphi\right\|_{L^{2}} .
$$

We use the following notation of pseudo-differential operators. For any symbol $a \in C^{\infty}\left(\mathbb{R}^{2 n}\right)$, we define the Weyl quantization of $a$ (at least formally) by

$$
\operatorname{Op}(a) u(x)=(2 \pi)^{-n} \iint e^{i(x-y) \cdot \xi} a((x+y) / 2, \xi) u(y) d y d \xi, \quad u \in \mathcal{S}\left(\mathbb{R}^{n}\right) .
$$

We set the symbol classes $S, S^{k, \ell}$ and $S(m, g)$ by

$$
\begin{aligned}
& S:=\left\{a \in C^{\infty}\left(\mathbb{R}^{2 n}\right)\left|\forall \alpha, \beta, \exists C_{\alpha \beta}:\right| \partial_{x}^{\alpha} \partial_{\xi}^{\beta} a(x, \xi) \mid \leqslant C_{\alpha \beta}\right\}, \\
& S^{k, \ell}:=\left\{a \in C^{\infty}\left(\mathbb{R}^{2 n}\right)\left|\forall \alpha, \beta, \exists C_{\alpha \beta}:\right| \partial_{x}^{\alpha} \partial_{\xi}^{\beta} a(x, \xi) \mid \leqslant C_{\alpha \beta}\langle x\rangle^{k-|\alpha|}\langle\xi\rangle^{\ell-|\beta|}\right\}, \\
& S(m, g):=\left\{a \in C^{\infty}\left(\mathbb{R}^{2 n}\right) \mid \text { for vector fields }: X_{1}, X_{2}, \ldots, X_{k}, \exists C\right. \text { such that } \\
& \left.\quad\left|X_{1} X_{2} \ldots X_{k} a(x, \xi)\right| \leqslant C m(x, \xi) g\left(X_{1}, X_{1}\right)^{\frac{1}{2}} g\left(X_{2}, X_{2}\right)^{\frac{1}{2}} \cdots g\left(X_{k}, X_{k}\right)^{\frac{1}{2}}\right\},
\end{aligned}
$$

where $g$ is a slowing varying metric and $m$ is a $g$-continuous function (see [Hör85, Section 18.4]). We denote the Poisson bracket of symbols $a$ and $b$ by $\{a, b\}=$ $\partial_{x} a \cdot \partial_{\xi} b-\partial_{\xi} a \cdot \partial_{x} b$.

The proofs of the following lemmas are standard, and we omit the proofs. 
Lemma 2.1. - Let $\left(x_{0}, \xi_{0}\right) \in \mathbb{R}^{2 n}$ with $\xi_{0} \neq 0$. Suppose that there exists $a \in S$ such that $a\left(x_{0}, \xi_{0}\right)>0$ and $\left\|\mathrm{Op}\left(a_{h}\right) u\right\|_{L^{2}}=O\left(h^{k+\varepsilon}\right)$ for some $\varepsilon>0$, where $a_{h}(x, \xi)=$ $a(x, h \xi)$. Then $u \in H^{k}$ microlocally at $\left(x_{0}, \xi_{0}\right)$.

Lemma 2.2. - Let $k, \ell \in \mathbb{R}$. Assume $a_{j} \in S^{k, \ell}$ is a bounded sequence in $S^{k, \ell}$ and $a_{j} \rightarrow 0$ in $S^{k+\delta, \ell+\delta}$ for some $\delta>0$. Then, for each $s, t \in \mathbb{R}$ and $u \in H^{s, t}$

$$
\left\|\mathrm{Op}\left(a_{j}\right) u\right\|_{H^{s-\ell, t-k}} \rightarrow 0 \quad \text { as } j \rightarrow \infty \text {. }
$$

\section{Proof of Theorem 1.3}

By the basic criterion for the essential self-adjointness ([RS80, Theorem VII.3]), it is sufficient to show

$$
\operatorname{Ker}\left(P^{*} \pm i\right)=\{0\}
$$

to prove Theorem 1.3. Since $D(P)=C_{c}^{\infty}\left(\mathbb{R}^{n}\right)$, we have $D\left(P^{*}\right)=\left\{u \in L^{2}\left(\mathbb{R}^{n}\right) \mid P u \in\right.$ $\left.L^{2}\left(\mathbb{R}^{n}\right)\right\}$ where $P$ acts on $u$ in the distribution sense. We hence show:

$$
(P \pm i) u=0 \text { in } \mathcal{D}^{\prime}\left(\mathbb{R}^{n}\right) \text { for } u \in L^{2}\left(\mathbb{R}^{n}\right) \text { implies } u=0 .
$$

We only consider "+" case. The "-" case is similarly handled. Moreover, we note if $u$ satisfies $(P+i) u=0$ and $u \in H^{\frac{m-1}{2},-\frac{1}{2}}\left(\mathbb{R}^{n}\right)$, then $u=0$ follows from a simple argument in [Vas20]. Namely, we take a real-valued function $\psi \in C_{c}^{\infty}(\{t \in \mathbb{R} \mid t \leqslant 2\})$ such that $\psi(t)=1$ for $t \leqslant 1$ and set $\psi_{R}(x, \xi)=\psi(\langle x\rangle / R) \psi(\langle\xi\rangle / R)$. Then we have

$$
2 i\|u\|_{L^{2}}^{2}=(P u, u)_{L^{2}}-(u, P u)_{L^{2}}=\lim _{R \rightarrow \infty}\left(\left[\operatorname{Op}\left(\psi_{R}\right), P\right] u, u\right)_{L^{2}} .
$$

We note that $\left[\mathrm{Op}\left(\psi_{R}\right), P\right]$ is uniformly bounded in $\mathrm{Op} S^{m-1,-1}$ and converges to 0 in $\mathrm{Op} S^{m-1+\delta,-1+\delta}$ as $R \rightarrow \infty$ for any $\delta>0$. We obtain $u=0$ by using Lemma 2.2 . Thus, in order to prove Theorem 1.3, it suffices to prove

Proposition 3.1. - If $u \in L^{2}\left(\mathbb{R}^{n}\right)$ satisfies $(P+i) u=0$, then $u \in H^{\frac{m-1}{2},-\frac{1}{2}}$.

The proof of Proposition 3.1 is divided into two parts. In Subsection 3.1, we prove the local smoothness of $u$. In Subsection 3.2, using the local smoothness of $u$, we prove weighted Sobolev properties of $u$.

\subsection{Local regularity}

The main result of this subsection is the following proposition. We note that we need the null non-trapping condition only for this proposition.

Proposition 3.2. - If $u \in L^{2}\left(\mathbb{R}^{n}\right)$ satisfies $(P+i) u=0$, then $u \in C^{\infty}\left(\mathbb{R}^{n}\right)$.

Proof. - It suffices to prove $u \in H_{\mathrm{loc}}^{k}\left(\mathbb{R}^{n}\right)$ for any $k>0$. We use the contradiction argument. Suppose $u \notin H_{\text {loc }}^{k}\left(\mathbb{R}^{n}\right)$ with some $k$. By Lemma 2.1, there exist $\left(x_{0}, \xi_{0}\right) \in$ $\mathbb{R}^{n} \times \mathbb{R}^{n}$ with $\xi_{0} \neq 0, C>0$, and a sequence $\left\{h_{\ell}\right\} \subset(0,1]$ such that for any $a \in S$ with $a\left(x_{0}, \xi_{0}\right)=1$,

$$
h_{\ell} \rightarrow 0 \text { as } \ell \rightarrow \infty \text {, and }\left\|\mathrm{Op}\left(a_{h_{\ell}, m}\right) u\right\| \geqslant C h_{\ell}^{\frac{k}{m-1}+1},
$$


where $a_{h, m}(x, \xi)=a\left(x, h^{\frac{1}{m-1}} \xi\right)$. We may assume $\left(x_{0}, \xi_{0}\right) \in p_{m}^{-1}(0)$ since $u$ is smooth microlocally in $\mathbb{R}^{2 n} \backslash p_{m}^{-1}(\{0\})$. Now we use the following proposition.

Proposition 3.3. - There exists a family of bounded operators

$$
\{F(h, t)\}_{0<h \leqslant 1, t \geqslant 0} \text { on } L^{2}\left(\mathbb{R}^{n}\right)
$$

such that

(1) $F(h, 0)=\mathrm{Op}\left(\psi_{h}\right)^{2}=\mathrm{Op}\left(\psi_{h}\right)^{*} \mathrm{Op}\left(\psi_{h}\right)$, where $\psi_{h}$ satisfies conditions: $\psi_{h}\left(x_{0}\right.$, $\left.h^{-\frac{1}{m-1}} \xi_{0}\right) \geqslant 1$, and for any $\alpha, \beta \in \mathbb{Z}_{+}^{n}$,

$$
\left|\partial_{x}^{\alpha} \partial_{\xi}^{\beta} \psi_{h}(x, \xi)\right| \leqslant C_{\alpha \beta} h^{\frac{|\beta|}{m-1}}\langle x\rangle^{-|\alpha|} .
$$

(2) There exists $C>0$ such that for $0<h \leqslant 1$,

$$
\|F(h, t)\|_{B\left(L^{2}\right)} \leqslant C\langle t\rangle h^{(-m+2) /(m-1)}, \quad t \geqslant 0 .
$$

(3) There exists $R(h, t) \in B\left(L^{2}\left(\mathbb{R}^{n}\right)\right)$ such that

$$
\begin{aligned}
& \frac{d}{d t} F(h, t)+i[P, F(h, t)] \geqslant-R(h, t), \quad t \geqslant 0, \\
& \sup _{t \geqslant 0}\langle t\rangle^{-1}\|R(h, t)\|_{B\left(L^{2}\right)}=O\left(h^{\infty}\right) \quad \text { as } h \rightarrow 0 .
\end{aligned}
$$

Proposition 3.3 can be proved similarly as [Nak05, Lemma 9]. For the completeness, we give a proof of Proposition 3.3 in the Appendix B. Now we set $u(t, x):=e^{-t} u(x)$. Then $u(t, x)$ satisfies

$$
i \partial_{t} u(t, x)-P u(t, x)=0,\|u(t)\|_{L^{2}\left(\mathbb{R}^{n}\right)} \leqslant e^{-t}\|u\|_{L^{2}\left(\mathbb{R}^{n}\right)},
$$

where the first equality is in the distributional sense. Setting $a(x, \xi)=\psi_{h}\left(x, h^{-\frac{1}{m-1}} \xi\right)$, then we note $a \in S, a_{h, m}=\psi_{h}$ and $a\left(x_{0}, \xi_{0}\right)=1$. We set $F_{\ell}(t)=F\left(h_{\ell}, t\right)$. Then, we have

$$
\begin{aligned}
C h_{\ell}^{\frac{2 k}{m-1}+2} & \leqslant\left\|\mathrm{Op}\left(\psi_{h_{\ell}}\right) u\right\|^{2}=\left(u, F_{\ell}(0) u\right) \\
& =\left(u(t), F_{\ell}(t) u(t)\right)-\int_{0}^{t} \frac{d}{d s}\left(u(s), F_{\ell}(s) u(s)\right) d s \\
& =\left(u(t), F_{\ell}(t) u(t)\right)-\int_{0}^{t}\left(u(s),\left(\frac{d F_{\ell}}{d s}(s)+i\left[P, F_{\ell}(s)\right]\right) u(s)\right) d s \\
& \leqslant C h_{\ell}^{\frac{-m+2}{m-1}}\langle t\rangle e^{-2 t}\|u\|^{2}+O\left(h_{\ell}^{\infty}\right) \cdot\|u\|^{2} \int_{0}^{t} e^{-2 s}\langle s\rangle d s
\end{aligned}
$$

where all the inner products and norms here are in $L^{2}\left(\mathbb{R}^{n}\right)$, and $O\left(h_{\ell}^{\infty}\right)$ is uniformly in $t$. Now, we take $t=h_{\ell}^{-1}$ then we conclude a contradiction. Thus, we obtain $u \in H_{\mathrm{loc}}^{k}\left(\mathbb{R}^{n}\right)$ for any $k>0$. This completes the proof of Proposition 3.2.

\subsection{Uniform regularity outside a compact set}

In this subsection, we prove a priori sub-elliptic estimates near infinity. The following estimates are based on the radial points estimates in [Mel94], where the radial points estimates are used for scattering theory on scattering manifolds. By 
the classical propagation of singularities, the singularities of a solution to $P u=0$ (provided $P$ is real-valued real principal type) propagate along the Hamilton flow associated with $p$. At points where the Hamilton vector field vanishes, we may use the so-called radial points, which implies $u$ is rapidly decaying at a radial source if $u$ has a threshold regularity at the radial source.

In our case, the radial points estimates are analogous to the Mourre estimate microlocally near outgoing or incoming regions, which is used commonly in scattering theory. We give a self-contained proof of the radial point estimate based on an explicit construction of escaping functions. We note the operator theoretical framework of the Mourre theory is not applicable here since we do not have the self-adjointness of $P$ at this point.

We set

$$
P=P_{0}+Q, \quad P_{0}=p_{0}\left(D_{x}\right), \quad Q=\mathrm{Op}(q),
$$

where

$$
q(x, \xi)=p(x, \xi)-p_{0}(\xi) \in S^{m,-\mu}, V(x, \xi)=p(x, \xi)-p_{m}(x, \xi) \in S^{m-1,-\mu} .
$$

We use the following smooth cut-off functions: Let $\chi \in C^{\infty}(\mathbb{R})$ be such that

$$
\chi(t)=\left\{\begin{array}{ll}
1 & \text { if } t \leqslant 1, \\
0 & \text { if } t \geqslant 2,
\end{array} \quad 0 \leqslant \chi(t) \leqslant 1, \quad \chi^{\prime}(t) \leqslant 0 \quad \text { for } t \in \mathbb{R},\right.
$$

and supp $\chi^{\prime} \Subset(1,2)$. We write $\bar{\chi}(t)=1-\chi(t)$, and

$$
\chi_{M}(x)=\chi(|x| / M), \quad \bar{\chi}_{M}(x)=\bar{\chi}(|x| / M), \quad x \in \mathbb{R}^{n},
$$

with $M>0$. A main result of this subsection is the following theorem.

Theorem 3.4. - Let $\gamma>0$ and $z \in \mathbb{C} \backslash \mathbb{R}$. There is $M>0$ such that if $\varphi \in L^{2}\left(\mathbb{R}^{n}\right),(P-z) \varphi \in \mathcal{S}\left(\mathbb{R}^{n}\right)$ and $\chi_{M}(x) \varphi \in C^{\infty}\left(\mathbb{R}^{n}\right)$, then $\varphi \in H^{k+1-m / 2,-\gamma} \cap$ $H^{k+1 / 2,-\gamma-1 / 2}$ for any $k \in \mathbb{R}$.

Now we show Proposition 3.1 follows from Theorem 3.4.

Proof of Proposition 3.1. - Suppose that $u \in L^{2}\left(\mathbb{R}^{n}\right)$ satisfies $(P+i) u=0$. By Proposition 3.2, we have $u \in C^{\infty}\left(\mathbb{R}^{n}\right)$. In particular, we have $\chi_{M}(x) \varphi \in C^{\infty}\left(\mathbb{R}^{n}\right)$ for any $M \geqslant 1$. Taking $\gamma=1 / 2$ and $k=m-1$, we obtain $\varphi \in H^{m / 2,-1 / 2} \subset H^{(m-1) / 2,-1 / 2}$. This completes the proof of Proposition 3.1.

Thus it remains to prove Theorem 3.4. In the following, we assume $\operatorname{Im} z>0$ without loss of generality. We may also assume $0<\gamma<\min (1 / 4, \mu / 2)$.

Weight functions: We choose $\rho(t) \in C^{\infty}(\mathbb{R})$ such that

$$
\rho(t)=\left\{\begin{array}{ll}
0 & \text { if } t \leqslant 0, \\
1 & \text { if } t \geqslant 1 / 8,
\end{array} \quad 0 \leqslant \rho(t) \leqslant 1, \quad \rho^{\prime}(t) \geqslant 0 \quad \text { for } t \in \mathbb{R} .\right.
$$

For $\delta \in(1 / 2,7 / 8)$, we set

$$
\rho_{+}^{\delta}(t)=\rho(t-\delta), \quad \rho_{-}^{\delta}(t)=1-\rho(t+1-\delta), \quad \rho_{0}^{\delta}(t)=1-\rho_{+}^{\delta}(t)-\rho_{-}^{\delta}(t),
$$


for $t \in \mathbb{R}$. We use the following notation:

$$
\widehat{x}=\frac{x}{|x|}, \quad v(\xi)=\partial_{\xi} p_{0}(\xi), \quad \widehat{v}(\xi)=\frac{v(\xi)}{|v(\xi)|}, \quad \eta=\eta(x, \xi)=\widehat{x} \cdot \widehat{v}(\xi) .
$$

Then we set

$$
b^{\delta}(x, \xi)=\left(\rho_{-}^{\delta}(\eta)|x|^{\gamma}+\rho_{0}^{\delta}(\eta)+\rho_{+}^{\delta}(\eta)|x|^{-\gamma}\right) e^{-\gamma \eta},
$$

which is defined for $x, \xi \in \mathbb{R}^{n} \backslash\{0\}$. We introduce cut-off functions and set

$$
b_{M, \nu}^{\delta}(x, \xi)=b^{\delta}(x, \xi) \bar{\chi}_{M}(x) \bar{\chi}_{\nu}(\xi), \quad x, \xi \in \mathbb{R}^{n} .
$$

with $M, \nu>0$. We also write

$$
\begin{aligned}
& \Omega_{1}(M, \nu)=\{(x, \xi)|M \leqslant| x|\leqslant 2 M,| \xi \mid \geqslant \nu\}, \\
& \Omega_{2}(M, \nu)=\{(x, \xi)|| x|\geqslant M, \nu \leqslant| \xi \mid \leqslant 2 \nu\} .
\end{aligned}
$$

We set a Riemannian metric $g$ on $\mathbb{R}^{2 n}$ by

$$
g=\frac{d x^{2}}{\langle x\rangle^{2}}+\frac{d \xi^{2}}{\langle\xi\rangle^{2}} .
$$

The next lemma is a key of the proof of Theorem 3.4.

Lemma 3.5. - Let $1 / 2<\delta<\widetilde{\delta}<7 / 8, k \in \mathbb{R}, 0<\widetilde{M}<M, 0<\widetilde{\nu}<\nu$, and write

$$
B=\mathrm{Op}\left(b_{M, \nu}^{\delta}\right), \quad \widetilde{B}=\mathrm{Op}\left(b_{\tilde{M}, \tilde{\nu}}^{\tilde{\delta}}\right) .
$$

If $\widetilde{M}$ is sufficiently large, then: There are pseudodifferential operators $S=\operatorname{Op}\left(f_{1}\right)$, $T=\mathrm{Op}\left(f_{2}\right)$ such that $f_{1}, f_{2} \in S(1, g)$ and $\operatorname{supp}\left[f_{1}\right] \subset \Omega_{1}(M, \nu)$, supp $\left[f_{2}\right] \subset$ $\Omega_{2}(M, \nu) ;$ If $\varphi \in \mathcal{S}^{\prime}, \widetilde{B} \varphi \in H^{k-1+m / 2,-1 / 2}, B(P-z) \varphi \in H^{k-(m-1) / 2,1 / 2}, S \varphi \in$ $H^{k+(m-1) / 2}$ and $T \varphi \in L^{2}$ then

$$
B \varphi \in H^{k} \cap H^{k+(m-1) / 2,-1 / 2} .
$$

Moreover, For any $N>0$ and $k \geqslant 0$ there is $C>0$ such that

$$
\begin{aligned}
& \|B \varphi\|_{H^{k+(m-1) / 2,-1 / 2}}^{2}+(\operatorname{Im} z)\|B \varphi\|_{H^{k}}^{2} \\
& \leqslant C\left(\|B(P-z) \varphi\|_{H^{k-(m-1) / 2,1 / 2}+\|\widetilde{B} \varphi\|_{H^{k-1+m / 2,-1}}^{2}}^{2}\right. \\
& \left.\quad+\|S \varphi\|_{H^{k+(m-1) / 2}}^{2}+\|T \varphi\|_{L^{2}}^{2}+\|\varphi\|_{H^{-N,-N}}^{2}\right) .
\end{aligned}
$$

Remark 3.6. - The constant $C$ in the lemma is independent of $\varphi$ and $z \in \mathbb{C} \backslash \mathbb{R}$. We note we assume $\widetilde{B} \varphi \in H^{k+(m-1) / 2,-1 / 2}$ for technical reasons, though only the norm of $\widetilde{B} \varphi$ in $H^{k-1+\frac{m}{2},-1}$ appears in the RHS of (3.1).

Theorem 3.4 follows from Lemma 3.5.

Proof of Theorem 3.4. - For $j=0,1,2, \ldots$, we choose $\nu_{j}$ and $\widetilde{\nu}_{j}$ so that

$$
0<\widetilde{\nu}_{0}<\nu_{0}=\widetilde{\nu}_{1}<\nu_{1}=\widetilde{\nu}_{2}<\nu_{2}=\cdots<\delta_{0}<\infty
$$

with an arbitrarily fixed $\delta_{0}>0$. We then choose $M_{j}$ and $\widetilde{M}_{j}$ so that the claim of Lemma 3.5 holds with $k=j / 2, M=M_{j}, \widetilde{M}=\widetilde{M}_{j}$ and

$$
0<\widetilde{M}_{0}<M_{0}=\widetilde{M}_{1}<M_{1}=\widetilde{M}_{2}<M_{2}=\cdots .
$$


We also set $\delta_{j}=\left(1+2^{-j}\right) / 4$ and $\widetilde{\delta}_{j}=\delta_{j-1}=\left(1+2 \cdot 2^{-j}\right) / 4$ for $j=0,1,2, \ldots$ We write $B_{j}=\operatorname{Op}\left(b_{M_{j}, \nu_{j}}^{\delta_{j}}\right), \widetilde{B}_{j}=\operatorname{Op}\left(b_{\tilde{M}_{j}, \tilde{\nu}_{j}}^{\tilde{\delta}_{j}}\right)=B_{j-1}$.

Suppose $\varphi \in L^{2}$ and $(P-z) \varphi \in \mathcal{S}\left(\mathbb{R}^{n}\right)$. Then we note

$$
B_{j}(P-z) \varphi \in \mathcal{S}\left(\mathbb{R}^{n}\right)
$$

At first, we have $\widetilde{B}_{0} \varphi \in H^{0,-\gamma} \subset H^{0,-1 / 2}$. By Lemma 3.5 with $k=1-m / 2$, we learn $\widetilde{B}_{1} \varphi=B_{0} \varphi \in H^{1-m / 2} \cap H^{1 / 2,-1 / 2}$, provided $S \varphi \in H^{1 / 2}$ and $T \varphi \in L^{2}$, which are satisfied under the assumptions of Theorem 3.4 (with $M_{0} \leqslant M$ ). Then we use Lemma 3.5 again with $k=(3-m) / 2$ to learn $\widetilde{B}_{2} \varphi=B_{1} \varphi \in H^{(3-m) / 2} \cap H^{1,-1 / 2}$. Iterating this procedure $2 k$-times, we arrive at

$$
B_{2 k} \varphi \in H^{k+1-m / 2} \cap H^{k+1 / 2,-1 / 2} .
$$

Note that conditions $S \varphi \in H^{k / 2+1 / 2}$ and $T \varphi \in L^{2}$ are satisfied since $\chi_{M}(x) \varphi \in$ $C^{\infty}\left(\mathbb{R}^{n}\right)$ and $\varphi \in L^{2}\left(\mathbb{R}^{n}\right)$. Now we use the first inclusion $B_{2 k} \varphi \in H^{k+1-m / 2}$. We recall, by the assumption, $\chi_{M} \varphi \in H^{k+1-m / 2}$, and this implies

$$
B_{2 k} \varphi+\chi_{M}(x) \varphi \in H^{k+1-m / 2} .
$$

Since

$$
b_{M, \nu}+\chi_{M}(x) \geqslant c_{0}\langle x\rangle^{-\gamma}, \quad|\xi| \geqslant 2 \nu,
$$

by the elliptic estimates (or the sharp Gårding inequality), we have $\varphi \in H^{k+1-m / 2,-\gamma}$. $\varphi \in H^{k+1 / 2,-\gamma-1 / 2}$ follows from $B_{2 k} \varphi \in H^{k+1 / 2,-1 / 2}$ by the same argument.

For the proof of Lemma 3.5, we compute the commutator of $B$ and $P$, and then use a commutator inequality. We write $b=b_{M, \nu}^{\delta}, \widetilde{b}=b_{\tilde{M}}^{\tilde{\delta}}, \rho_{*}^{\delta}=\rho_{*}$ and $\widetilde{\rho}_{*}=\rho_{*}^{\tilde{\delta}}$, where $*=+,-$, or 0 . The following lower bound for the Poisson bracket is crucial in the proof of Lemma 3.5.

Lemma 3.7. - Let $k, M$ and $\nu$ be as in Lemma 3.5. If $M$ is sufficiently large, there are symbols $f_{1}, f_{2} \in S(1, g)$ such that supp $\left[f_{1}\right] \subset \Omega_{1}(M, \nu)$, supp $\left[f_{2}\right] \subset \Omega_{2}(M, \nu)$, $f_{1}, f_{2} \geqslant 0, f_{2} \leqslant C\langle x\rangle^{-(1+\mu-2 \gamma) / 2} b$, and $\delta_{4}>0$ such that

$$
\left\{p,\langle\xi\rangle^{2 k} b^{2}\right\} \geqslant \delta_{4} \frac{|v|}{|x|}\langle\xi\rangle^{2 k} b^{2}-\langle\xi\rangle^{2 k+m-1} f_{1}^{2}-f_{2}^{2} .
$$

Proof. — We first note

$$
v \cdot \partial_{x} \eta=v \cdot \frac{\partial \widehat{x}}{\partial x} \widehat{v}=|v|\left\langle\widehat{v},\left(\frac{E}{|x|}-\frac{x \otimes x}{|x|^{3}}\right) \widehat{v}\right\rangle=\frac{|v|}{|x|}\left(1-\eta^{2}\right),
$$

where $E$ denotes the identity matrix. We also note

$$
\rho_{0}^{\prime}=-\rho_{+}^{\prime}-\rho_{-}^{\prime}, \quad \partial_{x}|x|=\widehat{x}, \quad v \cdot\left(\partial_{x}|x|\right)=|v| \widehat{v} \cdot \widehat{x}=|v| \eta .
$$


Using these, we compute:

$$
\begin{aligned}
& \left\{p_{0}, b\right\}=v \cdot \partial_{x} b \\
& =\left(v \cdot \partial_{x} \eta\right)\left\{\rho_{-}^{\prime}|x|^{\gamma}+\rho_{0}^{\prime}+\rho_{+}^{\prime}|x|^{-\gamma}-\gamma\left(\rho_{-}|x|^{\gamma}+\rho_{0}+\rho_{+}|x|^{-\gamma}\right)\right\} \\
& \times \bar{\chi}_{M}(x) \bar{\chi}_{\nu}(\xi) e^{-\gamma \eta}+\left(v \cdot \partial_{x}|x|\right)\left(\gamma \rho_{-}|x|^{\gamma-1}-\gamma \rho_{+}|x|^{-\gamma-1}\right) \bar{\chi}_{M}(x) \bar{\chi}_{\nu}(\xi) e^{-\gamma \eta} \\
& +\left(v \cdot \partial_{x}|x|\right)\left(\rho_{-}|x|^{\gamma}+\rho_{0}+\rho_{+}|x|^{-\gamma}\right) M^{-1} \bar{\chi}^{\prime}(|x| / M) \bar{\chi}_{\nu}(\xi) e^{-\gamma \eta} \\
& =\frac{|v|}{|x|}\left(1-\eta^{2}\right)\left\{\rho_{-}^{\prime}\left(|x|^{\gamma}-1\right)+\rho_{+}^{\prime}\left(|x|^{-\gamma}-1\right)-\gamma\left(\rho_{-}|x|^{\gamma}+\rho_{0}+\rho_{+}|x|^{-\gamma}\right)\right\} \\
& \times \bar{\chi}_{M}(x) \bar{\chi}_{\nu}(\xi) e^{-\gamma \eta}+\gamma \frac{|v|}{|x|}\left(\eta \rho_{-}|x|^{\gamma}-\eta \rho_{+}|x|^{-\gamma}\right) \bar{\chi}_{M}(x) \bar{\chi}_{\nu}(\xi) e^{-\gamma \eta}+r_{0},
\end{aligned}
$$

where

$$
r_{0}(x, \xi)=|v(\xi)| \eta(x, \xi) b^{\delta}(x, \xi) M^{-1} \bar{\chi}^{\prime}(|x| / M) \bar{\chi}_{\nu}(\xi),
$$

which is supported in $\Omega_{1}(M, \nu)$. We may suppose $M \geqslant 1$, and then

$$
\rho_{-}^{\prime}\left(|x|^{\gamma}-1\right) \leqslant 0, \quad \rho_{+}^{\prime}\left(|x|^{-\gamma}-1\right) \leqslant 0
$$

on the support of $b$. We also note

$$
\eta \rho_{-}(\eta) \leqslant(-7 / 8+\delta) \rho_{-}(\eta),-\eta \rho_{+}(\eta) \leqslant-\delta \rho_{+}(\eta),
$$

and

$$
\left(1-\eta^{2}\right) \rho_{0}(\eta) \geqslant \min \left(1-(\delta-1)^{2}, 1-(\delta+1 / 8)^{2}\right) \rho_{0}(\eta)
$$

We set

$$
\delta_{3}=\min \left(7 / 8-\delta, \delta, 1-(\delta-1)^{2}, 1-(\delta+1 / 8)^{2}\right)>0 .
$$

We substitute these inequality to the above formula on $\left\{p_{0}, b\right\}$ to learn

$$
\begin{aligned}
\left\{p_{0}, b\right\} & \leqslant-\gamma \delta_{3} \frac{|v|}{|x|}\left\{\rho_{0}+\rho_{-}|x|^{\gamma}+\rho_{+}|x|^{-\gamma}\right\} \bar{\chi}_{M}(x) \bar{\chi}_{\nu}(\xi) e^{-\gamma \eta}+r_{0} \\
& \leqslant-\delta_{3} \gamma \frac{|v|}{|x|} b(x, \xi)+r_{0}(x, \xi) .
\end{aligned}
$$

Then we have

$$
-\left\{p_{0}, b^{2}\right\}=-2 b\left\{p_{0}, b\right\} \geqslant 2 \delta_{3} \gamma \frac{|v|}{|x|} b^{2}-2 b r_{0} .
$$

This also implies

$$
-\left\{p_{0},\langle\xi\rangle^{2 k} b^{2}\right\}=-2 b\left\{p_{0}, b\right\}\langle\xi\rangle^{2 k} \geqslant 2 \delta_{3} \gamma \frac{|v|}{|x|}\langle\xi\rangle^{2 k} b^{2}-2\langle\xi\rangle^{2 k} b r_{0} .
$$

On the other hand, we have $\left\{q,\langle\xi\rangle^{2 k} b^{2}\right\} \in S\left(\langle x\rangle^{-\mu+2 \gamma-1}\langle\xi\rangle^{2 k+m-1}, g\right)$. We consider this function in more detail. We note, for any $\alpha, \beta \in \mathbb{Z}_{+}^{n}$,

$$
\left|\partial_{x}^{\alpha} \partial_{\xi}^{\beta} b^{\delta}(x, \xi)\right| \leqslant C_{\alpha \beta}|x|^{\gamma-|\alpha|}|\xi|^{-|\beta|}, \quad x, \xi \neq 0
$$


with some $C_{\alpha \beta}>0$. We also note

$$
\begin{aligned}
\{q, b\} & =\left\{q, b^{\delta}\right\} \bar{\chi}_{M}(x) \bar{\chi}_{\nu}(\xi)+b^{\delta}\left\{q, \bar{\chi}_{M}(x) \bar{\chi}_{\nu}(\xi)\right\} \\
& =\left\{q, b^{\delta}\right\} \bar{\chi}_{M}(x) \bar{\chi}_{\nu}(\xi)+r_{1}+r_{2},
\end{aligned}
$$

where

$$
r_{1}=b^{\delta}\left(\partial_{\xi} q\right) \cdot\left(\partial_{x} \bar{\chi}_{M}\right) \bar{\chi}_{\nu}(\xi), \quad r_{2}=-b^{\delta} \bar{\chi}_{M}(x)\left(\partial_{x} q\right) \cdot\left(\partial_{\xi} \bar{\chi}_{\nu}\right) .
$$

We observe that $r_{1}$ is supported in $\Omega_{1}(M, \nu)$, and $r_{1} \in S\left(\langle\xi\rangle^{m-1}, g\right) ; r_{2}$ is supported in $\Omega_{2}(M, \nu)$ and $r_{2} \in S\left(\langle x\rangle^{-1-\mu+\gamma}, g\right)$. Using (3.3), we have

$$
\begin{aligned}
\left|\left\{q, b^{\delta}\right\} \bar{\chi}_{M}(x) \bar{\chi}_{\nu}(\xi)\right| & \leqslant C\langle x\rangle^{-\mu+\gamma-1}\langle\xi\rangle^{m-1} \bar{\chi}_{M}(x) \bar{\chi}_{\nu}(\xi) \\
& \leqslant C^{\prime} M^{-(\mu-2 \gamma)} \frac{|v(\xi)|}{|x|} b(x, \xi)
\end{aligned}
$$

with some $C, C^{\prime}>0$. Moreover, since $q \in S^{m,-\mu}$, we have

$$
\left|\left\{q,\langle\xi\rangle^{2 k}\right\} b^{2}\right| \leqslant C^{\prime \prime}\langle\xi\rangle^{2 k+m-1}\langle x\rangle^{-\mu-1} \leqslant C^{\prime \prime \prime} M^{-\mu} \frac{|v(\xi)|}{|x|} b^{2}
$$

with $C^{\prime \prime}>0$ and $C^{\prime \prime \prime}>0$. Set $C_{1}=C^{\prime}+C^{\prime \prime \prime}$. Hence we learn

$$
\left\{q,\langle\xi\rangle^{2 k} b^{2}\right\} \geqslant-2 C_{1} M^{-(\mu-2 \gamma)} \frac{|v(\xi)|}{|x|}\langle\xi\rangle^{2 k} b^{2}+2\langle\xi\rangle^{2 k} b r_{1}+2\langle\xi\rangle^{2 k} b r_{2},
$$

uniformly in $M \geqslant 1$. Combining this with (3.2), we learn

$$
\left\{p,\langle\xi\rangle^{2 k} b^{2}\right\} \geqslant\left(2 \delta_{3} \gamma-2 C_{1} M^{-(\mu-2 \gamma)}\right)\langle\xi\rangle^{2 k} \frac{|v|}{|x|} b^{2}+2\langle\xi\rangle^{2 k} b\left(-r_{0}+r_{1}+r_{2}\right) .
$$

We recall $\gamma<\mu / 2$. We now choose $M$ so large that $2 C_{1} M^{-(\mu-2 \gamma)} \leqslant \delta_{3} \gamma$, and we obtain

$$
\left\{p,\langle\xi\rangle^{2 k} b^{2}\right\} \geqslant \delta_{3} \gamma \frac{|v|}{|x|}\langle\xi\rangle^{2 k} b^{2}+2\langle\xi\rangle^{2 k} b\left(-r_{0}+r_{1}+r_{2}\right) .
$$

We note supp $\left[-r_{0}+r_{1}\right] \Subset \Omega_{1}(M, \nu)$ and $-r_{0}+r_{1} \in S\left(\langle\xi\rangle^{m-1}, g\right)$, hence we can find $f_{1} \in S(1, g), f_{1} \geqslant 0, \operatorname{supp}\left[f_{1}\right] \subset \Omega_{1}(M, \nu)$ such that

$$
2\langle\xi\rangle^{2 k} b\left(-r_{0}+r_{1}\right) \geqslant-\langle\xi\rangle^{2 k+m-1} f_{1}^{2} .
$$

Similarly, since supp $\left[r_{2}\right] \Subset \Omega_{2}(M, \nu), r_{2} \in S\left(\langle x\rangle^{\gamma-\mu-1}, g\right)$, we can find $f_{2} \in S(1, g)$, $f_{2} \geqslant 0, \operatorname{supp}\left[f_{2}\right] \subset \Omega_{2}(M, \nu)$ such that

$$
2\langle\xi\rangle^{2 k} b r_{2} \geqslant-f_{2}^{2} \text { and } 0 \leqslant f_{2} \leqslant C\langle x\rangle^{-(1+\mu-2 \gamma) / 2} b .
$$

By setting $\delta_{4}=\delta_{3} \gamma$, we arrive at the conclusion of the Lemma 3.7.

We write

$$
B=\mathrm{Op}(b), \quad \widetilde{B}=\mathrm{Op}(\widetilde{b}), \quad \Lambda=\left\langle D_{x}\right\rangle^{(m-1) / 2}\langle x\rangle^{-1 / 2} .
$$

Lemma 3.8. - Under the above assumptions, there are pseudodifferential operators $S, T, U, V$ and a constant $\delta_{4}>0$ such that

$$
-i\left[P, B\left\langle D_{x}\right\rangle^{2 k} B\right] \geqslant \delta_{4} B\left\langle D_{x}\right\rangle^{k}|\Lambda|^{2}\left\langle D_{x}\right\rangle^{k} B-S^{*}\left\langle D_{x}\right\rangle^{2 k+m-1} S-T^{*} T-U-V,
$$

where 
(1) $S \in \mathrm{Op} S(1, g)$ and its symbol is supported in $\Omega_{1}(M, \nu)$;

(2) $T \in \mathrm{Op} S(1, g)$ and its symbol is supported in $\Omega_{2}(M, \nu)$;

(3) $U=\operatorname{Op}(u)$ with $u \in S\left(\langle x\rangle^{2 \gamma-2}\langle\xi\rangle^{2 k+m-2}, g\right)$ and or for any $\alpha, \beta \in \mathbb{Z}_{+}^{n}$,

$$
\left|\partial_{x}^{\alpha} \partial_{\xi}^{\beta} u(x, \xi)\right| \leqslant C\langle x\rangle^{-2-|\alpha|}\langle\xi\rangle^{2 k+m-2-|\beta|} \widetilde{b}(x, \xi)^{2} ;
$$

(4) $V \in \operatorname{Op} S\left(\langle x\rangle^{-\infty}\langle\xi\rangle^{-\infty}, g\right)$.

In the proof of Lemma 3.8, we use the following estimate:

Lemma 3.9. - Suppose $a$ be a symbol such that supp $[a] \subset \Omega^{\prime}$, where $\Omega^{\prime}=\left\{(x, \xi)|| x\left|\geqslant M^{\prime},\right| \xi \mid \geqslant \nu^{\prime}\right\}$ with $M^{\prime}>\widetilde{M}, \nu^{\prime}>\widetilde{\nu}$, and for any $\alpha, \beta \in \mathbb{Z}_{+}^{n}$,

$$
\left|\partial_{x}^{\alpha} \partial_{\xi}^{\beta} a(x, \xi)\right| \leqslant C_{\alpha \beta}\langle x\rangle^{2 \ell-|\alpha|}\langle\xi\rangle^{2 s-|\beta|} \widetilde{b}(x, \xi)^{2},
$$

where $s, \ell \in \mathbb{R}$. Then for any $N$, there is $C, C_{N}>0$ such that

$$
|\langle\varphi, \operatorname{Op}(a) \varphi\rangle| \leqslant C\|\widetilde{B} \varphi\|_{H^{s, \ell}}^{2}+C_{N}\|\varphi\|_{H^{-N,-N}}^{2}, \quad \varphi \in \mathcal{S}\left(\mathbb{R}^{n}\right) .
$$

Proof. - We note, for any $\alpha, \beta \in \mathbb{Z}_{+}^{n}$,

$$
\left|\partial_{x}^{\alpha} \partial_{\xi}^{\beta} \widetilde{b}(x, \xi)\right| \leqslant C_{\alpha \beta}^{\prime}\langle x\rangle^{-|\alpha|+2 \gamma(|\alpha+\beta|)}\langle\xi\rangle^{-|\beta|} \widetilde{b}(x, \xi), \quad(x, \xi) \in \Omega^{\prime} .
$$

We write $\widetilde{g}=\langle x\rangle^{-2+4 \gamma} d x^{2}+\langle x\rangle^{4 \gamma}\langle\xi\rangle^{-2} d \xi^{2}$. Using the above estimate and the assumption on $a$, and following the construction of parametrices for elliptic operators, we can construct a symbol $h(x, \xi) \in S(1, \widetilde{g})$ such that

$$
\operatorname{Op}(a)=\widetilde{B}\langle x\rangle^{\ell}\left\langle D_{x}\right\rangle^{s} \mathrm{Op}(h)\left\langle D_{x}\right\rangle^{s}\langle x\rangle^{\ell} \widetilde{B}+R,
$$

where $R \in S\left(\langle x\rangle^{-\infty}\langle\xi\rangle^{-\infty}, \widetilde{g}\right)$. The assertion follows from this since $\mathrm{Op}(h)$ is bounded in $L^{2}\left(\mathbb{R}^{n}\right)$.

Proof of Lemma 3.8. - By the standard pseudodifferential operator calculus, we can find $\tilde{f}_{1}, \widetilde{f}_{2}$ such that $\tilde{f}_{j} \in S(1, g)$, supp $\left[\tilde{f}_{j}\right] \subset \Omega_{j}(M, \nu), j=1,2$, and

$$
\begin{aligned}
& \operatorname{Op}\left(\langle\xi\rangle^{2 k+m-1} f_{1}^{2}\right) \leqslant \operatorname{Op}\left(\widetilde{f}_{1}\right)^{*}\left\langle D_{x}\right\rangle^{2 k+m-1} \mathrm{Op}\left(\widetilde{f}_{1}\right)+R_{1}, \\
& \operatorname{Op}\left(\langle x\rangle^{2 \gamma-1-\mu} f_{2}^{2}\right) \leqslant \operatorname{Op}\left(\tilde{f}_{2}\right)^{*} \mathrm{Op}\left(\widetilde{f}_{2}\right)+R_{2},
\end{aligned}
$$

where $R_{j}$ are smoothing operators. We set $S=\operatorname{Op}\left(\tilde{f}_{1}\right)$ and $T=\operatorname{Op}\left(\tilde{f}_{2}\right)$. We denote

$$
\zeta(x, \xi)=\left\{p,\langle\xi\rangle^{2 k} b^{2}\right\}-\delta_{4} \frac{|v|}{|x|}\langle\xi\rangle^{2 k} b^{2}+\langle\xi\rangle^{2 k+m-1} f_{1}^{2}+f_{2}^{2} \geqslant 0 .
$$

We note, by the construction, $\zeta(x, \xi) b^{\prime}(x, \xi)^{-2} \in S\left(\langle x\rangle^{-1}\langle\xi\rangle^{2 k+m-1}, g\right)$, where $b^{\prime}=b_{M^{\prime}, \nu^{\prime}}^{\delta^{\prime}}$ with $\widetilde{M}<M^{\prime}<M, \widetilde{\nu}<\nu^{\prime}<\nu$ and $\delta<\delta^{\prime}<\widetilde{\delta}$. Hence by the sharp Gårding inequality, we have

$$
\mathrm{Op}\left(\zeta\left(b^{\prime}\right)^{-2}\right) \geqslant-C\left\langle D_{x}\right\rangle^{k-1+m / 2}\langle x\rangle^{-2}\left\langle D_{x}\right\rangle^{k-1+m / 2}
$$

with some $C>0$. Then by the asymptotic expansion, we learn

$$
\mathrm{Op}(\zeta) \geqslant-C B^{\prime}\left\langle D_{x}\right\rangle^{k-1+m / 2}\langle x\rangle^{-2}\left\langle D_{x}\right\rangle^{k-1+m / 2} B^{\prime}-R_{3},
$$

where $R_{3} \in S\left(\langle x\rangle^{-3}\langle\xi\rangle^{2 k+m-3}, g\right)$, and the symbol is supported in supp [b'] modulo $\mathcal{S}\left(\mathbb{R}^{2 d}\right)$. Using Lemma 3.7, we can estimate $R_{3}$ and other error terms from below 
by $-C \widetilde{B}\left\langle D_{x}\right\rangle^{k-1+m / 2}\langle x\rangle^{-2}\left\langle D_{x}\right\rangle^{k-1+m / 2} \widetilde{B}$, modulo smoothing operators, and these will be included in $U$ to complete the proof.

Lemma 3.10. - For $\varphi \in \mathcal{S}\left(\mathbb{R}^{n}\right)$, the inequality (3.1) holds, where $S=\operatorname{Op}\left(f_{1}\right)$, $T=\mathrm{Op}\left(f_{2}\right), f_{1}, f_{2} \in S(1, g)$, and $\operatorname{supp}\left[f_{1}\right] \subset \Omega_{1}(M, \nu)$, supp $\left[f_{2}\right] \subset \Omega_{2}(M, \nu)$.

Proof. - We compute the commutator to obtain quadratic inequalities. For $\varphi \in$ $\mathcal{S}\left(\mathbb{R}^{n}\right)$, we have

$$
\begin{aligned}
& \left\langle\varphi,-i\left[P, B\left\langle D_{x}\right\rangle^{2 k} B\right] \varphi\right\rangle=\left\langle\varphi,-i\left[(P-z), B\left\langle D_{x}\right\rangle^{2 k} B\right] \varphi\right\rangle \\
& =-i\left(\left\langle\left\langle D_{x}\right\rangle^{k} B(P-\bar{z}) \varphi,\left\langle D_{x}\right\rangle^{k} B \varphi\right\rangle-\left\langle\left\langle D_{x}\right\rangle^{k} B \varphi,\left\langle D_{x}\right\rangle^{k} B(P-z) \varphi\right\rangle\right) \\
& =-i\left(\left\langle\left(\Lambda^{-1}\right)^{*}\left\langle D_{x}\right\rangle^{k} B(P-z) \varphi, \Lambda\left\langle D_{x}\right\rangle^{k} B \varphi\right\rangle\right. \\
& \left.\quad-\left\langle\Lambda\left\langle D_{x}\right\rangle^{k} B \varphi,\left(\Lambda^{-1}\right)^{*}\left\langle D_{x}\right\rangle^{k} B(P-z) \varphi\right\rangle\right)-2(\operatorname{Im} z)\left\|\left\langle D_{x}\right\rangle^{k} B \varphi\right\|^{2} \\
& \quad \leqslant 2\left\|\left(\Lambda^{-1}\right)^{*}\left\langle D_{x}\right\rangle^{k} B(P-z) \varphi\right\| \cdot\left\|\Lambda\left\langle D_{x}\right\rangle^{k} B \varphi\right\|-2(\operatorname{Im} z)\left\|\left\langle D_{x}\right\rangle^{k} B \varphi\right\|^{2} .
\end{aligned}
$$

Combining this with Lemma 3.8, we have

$$
\begin{aligned}
\delta_{4}\left\|\Lambda\left\langle D_{x}\right\rangle^{k} B \varphi\right\|^{2}+2(\operatorname{Im} z) & \left\|\left\langle D_{x}\right\rangle^{k} B \varphi\right\|^{2}-\left\langle\varphi,\left(S^{*}\left\langle D_{x}\right\rangle^{2 k+m-1} S+T^{*} T+U+V\right) \varphi\right\rangle \\
& \leqslant 2\left\|\left(\Lambda^{-1}\right)^{*}\left\langle D_{x}\right\rangle^{k} B(P-z) \varphi\right\| \cdot\left\|\Lambda\left\langle D_{x}\right\rangle^{k} B \varphi\right\| \\
& \leqslant \frac{\delta_{4}}{2}\left\|\Lambda\left\langle D_{x}\right\rangle^{k} B \varphi\right\|^{2}+\frac{4}{\delta_{4}}\left\|\left(\Lambda^{-1}\right)^{*}\left\langle D_{x}\right\rangle^{k} B(P-z) \varphi\right\|^{2} .
\end{aligned}
$$

Thus we have

$$
\begin{aligned}
& \frac{\delta_{4}}{2}\left\|\Lambda\left\langle D_{x}\right\rangle^{k} B \varphi\right\|^{2}+2(\operatorname{Im} z)\left\|\left\langle D_{x}\right\rangle^{k} B \varphi\right\|^{2} \\
& \quad \leqslant \frac{4}{\delta_{4}}\left\|\left(\Lambda^{-1}\right)^{*}\left\langle D_{x}\right\rangle^{k} B(P-z) \varphi\right\|^{2}+\left\langle\varphi,\left(S^{*}\left\langle D_{x}\right\rangle^{2 k+m-1} S+T^{*} T+U+V\right) \varphi\right\rangle .
\end{aligned}
$$

Now we note, by Lemma 3.9,

$$
\langle\varphi, U \varphi\rangle \leqslant C\|\widetilde{B} \varphi\|_{H^{k-1+m / 2,-1}}^{2}+C\|\varphi\|_{H^{-N,-N}}^{2}
$$

with any $N$. These imply (3.1) for $\varphi \in \mathcal{S}\left(\mathbb{R}^{n}\right)$.

We now extend Lemma 3.10 to more general $\varphi$ to prove Lemma 3.5. We choose $M^{\prime}$ and $\nu^{\prime}$ so that $\widetilde{M}<M^{\prime}<M, \widetilde{\nu}<\nu^{\prime}<\nu, \delta<\delta^{\prime}<\widetilde{\delta}$, and set

$$
b^{\prime}(x, \xi)=b_{M^{\prime}, \nu^{\prime}}^{\delta^{\prime}}(x, \xi), \quad B^{\prime}=\operatorname{Op}\left(b^{\prime}\right) .
$$

We write

$$
A_{\varepsilon}=\left\langle\varepsilon D_{x}\right\rangle^{-1} B, \quad \widetilde{A}_{\varepsilon}=\left\langle\varepsilon D_{x}\right\rangle^{-1} \widetilde{B}, \quad A_{\varepsilon}^{\prime}=\left\langle\varepsilon D_{x}\right\rangle^{-1} B^{\prime}
$$

and we denote their symbols by $a_{\varepsilon}, \widetilde{a}_{\varepsilon}$ and $a_{\varepsilon}^{\prime}$, respectively.

By the same computation as in the proof of Lemma 3.7, we have

$$
\left\{p,\langle\xi\rangle^{2 k}\left|a_{\varepsilon}\right|^{2}\right\} \geqslant \delta_{4} \frac{|v|}{|x|}\langle\xi\rangle^{2 k}\left|a_{\varepsilon}\right|^{2}-\langle\xi\rangle^{2 k+m-1} f_{1}^{2}-\langle x\rangle^{2 \gamma-1-\mu} f_{2}^{2},
$$


modulo $\mathcal{S}\left(\mathbb{R}^{n}\right)$-terms, where constants are independent of $\varepsilon$, and $f_{1}$ and $f_{2}$ are independent of $\varepsilon$. Then, as well as Lemma 3.8, we have

$$
\begin{aligned}
-i\left[P, A_{\varepsilon}^{*}\left\langle D_{x}\right\rangle^{2 k} A_{\varepsilon}\right] \\
\\
\quad \geqslant \delta_{4} A_{\varepsilon}^{*}\left\langle D_{x}\right\rangle^{k} \Lambda^{2}\left\langle D_{x}\right\rangle^{k} A_{\varepsilon}-S^{*}\left\langle D_{x}\right\rangle^{2 k+m-1} S-T^{*} T-U_{\varepsilon}-V_{\varepsilon},
\end{aligned}
$$

where the symbol of $U_{\varepsilon}$ has the property:

$$
\left|u_{\varepsilon}(x, \xi)\right| \leqslant C\langle x\rangle^{-2}\langle\xi\rangle^{2 k+m-2}\left|a_{\varepsilon}^{\prime}(x, \xi)\right|^{2},
$$

and symbols of $U_{\varepsilon}$ and $V_{\varepsilon}$ are bounded in the respective symbol classes. It follows that

$$
\left|\left\langle\varphi, U_{\varepsilon} \varphi\right\rangle\right| \leqslant C\left\|A_{\varepsilon}^{\prime} \varphi\right\|_{H^{k-1+m / 2,-1}}^{2}+C\|\varphi\|_{H^{-N,-N}}^{2}, \quad \varphi \in \mathcal{S}\left(\mathbb{R}^{n}\right),
$$

where the constant is independent of $\varepsilon$. Thus we have, as well as Lemma 3.10, for $\varphi \in \mathcal{S}\left(\mathbb{R}^{n}\right)$,

$$
\begin{aligned}
& \left\|A_{\varepsilon} \varphi\right\|_{H^{k+(m-1) / 2,-1 / 2}}^{2}+(\operatorname{Im} z)\left\|A_{\varepsilon} \varphi\right\|_{H^{k}}^{2} \\
& \qquad C\left(\left\|A_{\varepsilon}(P-z) \varphi\right\|_{H^{k-(m-1) / 2,1 / 2}}^{2}+\left\|A_{\varepsilon}^{\prime} \varphi\right\|_{H^{k-1+m / 2,-1}}^{2}\right. \\
& \left.\quad+\|S \varphi\|_{H^{k+(m-1) / 2}}^{2}+\|T \varphi\|_{L^{2}}^{2}\right)+C_{N}\|\varphi\|_{H^{-N,-N}}^{2},
\end{aligned}
$$

with any $N$, where $C$ and $C_{N}$ are independent of $\varepsilon \in(0,1]$.

Lemma 3.11. - Suppose that $\varphi \in \mathcal{S}^{\prime}\left(\mathbb{R}^{n}\right)$ satisfies $\widetilde{B} \varphi \in H^{k-1+m / 2,-1 / 2}$,

$$
A_{\varepsilon}(P-z) \varphi \in H^{k-(m-1) / 2,1 / 2}, S \varphi \in H^{k+(m-1) / 2} \text { and } T \varphi \in L^{2} .
$$

Then $A_{\varepsilon} \varphi \in H^{k+(m-1) / 2,-1 / 2} \cap H^{m}$ and (3.5) holds.

Proof. — We set, for $L \gg 0$,

$$
X_{L}=\chi_{L}(x) \chi_{L}\left(D_{x}\right)
$$

We first note $\left\|X_{L} \psi-\psi\right\|_{H^{s, \ell}} \rightarrow 0$ as $L \rightarrow \infty$, provided $\psi \in H^{s, \ell}$. We also note $\psi \in H^{s, \ell}$ if and only if $\lim _{L \rightarrow \infty}\left\|X_{L} \psi\right\|_{H^{s, \ell}}<\infty$.

We observe that the symbol of $\left[X_{L}, A_{\varepsilon}\right]$ is bounded by $C\langle x\rangle^{-1}\langle\xi\rangle^{-1} a_{\varepsilon}^{\prime}(x, \xi)$, modulo $\mathcal{S}\left(\mathbb{R}^{2 d}\right)$-terms, uniformly in $L$, and also it converges to 0 locally uniformly as $L \rightarrow \infty$. These imply

$$
\begin{aligned}
\lim _{L \rightarrow \infty}\left\|X_{L} A_{\varepsilon} \psi\right\|_{H^{s, \ell}} & \leqslant \lim _{L \rightarrow \infty}\left(\left\|A_{\varepsilon} X_{L} \psi\right\|_{H^{s, \ell}}+\left\|\left[X_{L}, A_{\varepsilon}\right] \psi\right\|_{H^{s, \ell}}\right) \\
& \leqslant \underline{L \rightarrow \infty}\left\|A_{\varepsilon} X_{L} \psi\right\|_{H^{s, \ell}}
\end{aligned}
$$

with any $N$, provided $\widetilde{B} \psi \in H^{s-1, \ell-1}$. In particular, since we assume

$$
\begin{gathered}
\widetilde{B} \varphi \in H^{k-1+m / 2,-1 / 2}, \\
\lim _{L \rightarrow \infty}\left(\left\|X_{L} A_{\varepsilon} \varphi\right\|_{H^{k+(m-1) / 2,-1 / 2}}^{2}+\left\|X_{L} A_{\varepsilon} \varphi\right\|_{H^{k}}^{2}\right) \\
\leqslant \varliminf_{L \rightarrow \infty}\left(\left\|A_{\varepsilon} X_{L} \varphi\right\|_{H^{k+(m-1) / 2,-1 / 2}}^{2}+\left\|A_{\varepsilon} X_{L} \varphi\right\|_{H^{k}}^{2}\right) .
\end{gathered}
$$


By the same argument, using $\widetilde{B} \varphi \in H^{k-1+m / 2,-1 / 2}$, we learn

$$
\varlimsup_{L \rightarrow \infty}\left\|A_{\varepsilon}(P-z) X_{L} \varphi\right\|_{H^{k-(m-1) / 2,1 / 2}}^{2} \leqslant\left\|A_{\varepsilon}(P-z) \varphi\right\|_{H^{k-(m-1) / 2,1 / 2}}^{2} .
$$

We have similar estimates for $\|S \varphi\|_{H^{k+(m-1) / 2}}$ and $\|T \varphi\|_{L^{2}}$. Concerning the estimate for $\left\|A_{\varepsilon}^{\prime} \varphi\right\|_{H^{k-1+m / 2,-1}}$, we use the fact that $\widetilde{B} \varphi \in H^{k-1+m / 2,-1 / 2}$ to obtain

$$
\varlimsup_{L \rightarrow \infty}\left\|A_{\varepsilon}^{\prime} X_{L} \varphi\right\|_{H^{k-1+m / 2,-1}}^{2} \leqslant\left\|A_{\varepsilon}^{\prime} \varphi\right\|_{H^{k-1+m / 2,-1}}^{2} .
$$

Combining these with (3.5) for $X_{L} \varphi$, we learn

$$
\begin{aligned}
\lim _{L \rightarrow \infty} & \left(\left\|X_{L} A_{\varepsilon} \varphi\right\|_{H^{k+(m-1) / 2,-1 / 2}}^{2}+\left\|X_{L} A_{\varepsilon} \varphi\right\|_{H^{k}}^{2}\right) \\
\leqslant & \varlimsup_{L \rightarrow \infty}\left(C \left(\left\|A_{\varepsilon}(P-z) X_{L} \varphi\right\|_{H^{k-(m-1) / 2,1 / 2}}^{2}+\left\|A_{\varepsilon}^{\prime} X_{L} \varphi\right\|_{H^{k-1+m / 2,-1}}^{2}\right.\right. \\
& \left.\left.+\left\|S X_{L} \varphi\right\|_{H^{k+(m-1) / 2}}^{2}+\left\|T X_{L} \varphi\right\|_{L^{2}}^{2}\right)+C_{N}\left\|X_{L} \varphi\right\|_{H^{-N,-N}}^{2}\right) \\
\leqslant & C\left(\left\|A_{\varepsilon}(P-z) \varphi\right\|_{H^{k-(m-1) / 2,1 / 2}}^{2}+\left\|A_{\varepsilon}^{\prime} \varphi\right\|_{H^{k-1+m / 2,-1}}^{2}\right. \\
& \left.+\|S \varphi\|_{H^{k+(m-1) / 2}}^{2}+\|T \varphi\|_{L^{2}}^{2}\right)+C_{N}^{\prime}\|\varphi\|_{H^{-N,-N}}^{2}
\end{aligned}
$$

and this implies the assertion.

Proof of Lemma 3.5. - It remains to take the limit $\varepsilon \rightarrow 0$ in (3.5). We note

$$
\begin{aligned}
\left\|A_{\varepsilon} \varphi\right\|_{H^{s, \ell}} & =\left\|\left\langle D_{x}\right\rangle^{s}\langle x\rangle^{\ell}\left\langle\varepsilon D_{x}\right\rangle^{-1} B \varphi\right\|_{L^{2}} \\
& =\left\|\left\langle\varepsilon D_{x}\right\rangle^{-1}\left\langle D_{x}\right\rangle^{s}\langle x\rangle^{\ell} B \varphi+\left\langle D_{x}\right\rangle^{s}\left[\langle x\rangle^{\ell},\left\langle\varepsilon D_{x}\right\rangle^{-1}\right] B \varphi\right\|_{L^{2}},
\end{aligned}
$$

and hence

$$
\left\|\left\langle\varepsilon D_{x}\right\rangle^{-1}\left\langle D_{x}\right\rangle^{s}\langle x\rangle^{\ell} B \varphi\right\|_{L^{2}} \leqslant\left\|A_{\varepsilon} \varphi\right\|_{H^{s, \ell}}+C\|B \varphi\|_{H^{s-1, \ell-1}} .
$$

Thus we have

$$
\|B \varphi\|_{H^{s, \ell}} \leqslant \varliminf_{\varepsilon \rightarrow 0}\left\|A_{\varepsilon} \varphi\right\|_{H^{s, \ell}}+C\|B \varphi\|_{H^{s-1, \ell-1}} .
$$

We note this holds without assuming $B \varphi \in H^{s, \ell}$, and if the right hand side is finite, we obtain $B \varphi \in H^{s, \ell}$.

By the same argument, we also have

$$
\begin{aligned}
& \varlimsup_{\varepsilon \rightarrow 0}\left\|A_{\varepsilon}(P-z) \varphi\right\|_{H^{k-(m-1) / 2,1 / 2}} \\
& \quad \leqslant\|B(P-z) \varphi\|_{H^{k-(m-1) / 2,1 / 2}}+C\|B(P-z) \varphi\|_{H^{k-(m+1) / 2,-1 / 2}} \\
& \quad \leqslant(1+C)\|B(P-z) \varphi\|_{H^{k-(m-1) / 2,1 / 2}}
\end{aligned}
$$

and similarly,

$$
\varlimsup_{\varepsilon \rightarrow 0}\left\|A_{\varepsilon}^{\prime} \varphi\right\|_{H^{k-1+m / 2,-1}} \leqslant C^{\prime}\left\|B^{\prime} \varphi\right\|_{H^{k-1+m / 2,-1}} .
$$


Substituting these to (3.5), we have

$$
\begin{aligned}
& \|B \varphi\|_{H^{k+(m-1) / 2,-1 / 2}}^{2}+(\operatorname{Im} z)\|B \varphi\|_{H^{k}}^{2} \\
& \leqslant \lim _{\varepsilon \rightarrow 0}\left(\left\|A_{\varepsilon} \varphi\right\|_{H^{k+(m-1) / 2,-1 / 2}}^{2}+C\|B \varphi\|_{H^{k+(m-3) / 2,-3 / 2}}^{2}\right. \\
& \left.+(\operatorname{Im} z)\left(\left\|A_{\varepsilon} \varphi\right\|_{H^{k}}^{2}+C\|B \varphi\|_{H^{k-1,-1}}^{2}\right)\right) \\
& \leqslant \varlimsup_{\varepsilon \rightarrow 0} C\left(\left\|A_{\varepsilon}(P-z) \varphi\right\|_{H^{k-(m-1) / 2,1 / 2}}^{2}+\left\|\widetilde{A}_{\varepsilon} \varphi\right\|_{H^{k-1+m / 2,-1}}^{2}+\|B \varphi\|_{H^{k+(m-3) / 2,-1}}^{2}\right) \\
& +C\left(\|B \varphi\|_{H^{k-1,-1}}^{2}+\|S \varphi\|_{H^{k+(m-1) / 2}}^{2}+\|T \varphi\|_{L^{2}}^{2}\right)+C_{N}\|\varphi\|_{H^{-N,-N}}^{2} \\
& \leqslant C^{\prime}\left(\|B(P-z) \varphi\|_{H^{k-(m-1) / 2,1 / 2}}^{2}+\|\widetilde{B} \varphi\|_{H^{k-1+m / 2,-1}}^{2}\right. \\
& \left.+\|S \varphi\|_{H^{k+(m-1) / 2}}^{2}+\|T \varphi\|_{L^{2}}^{2}\right)+C_{N}\|\varphi\|_{H^{-N,-N}}^{2},
\end{aligned}
$$

and this completes the proof of Lemma 3.5.

\section{Appendix A. Estimates for the classical trajectories}

In this section, we prove estimates on the classical trajectories which are used in the proof of Proposition 3.3. First, we show a classical Mourre estimate which implies the pseudo-convexity of $\mathbb{R}^{n}$ with respect to $P$. We note

$$
(y(t, x, \lambda \xi), \eta(t, x, \lambda \xi))=\left(y\left(\lambda^{m-1} t, x, \xi\right), \lambda \eta\left(\lambda^{m-1} t, x, \xi\right)\right) \quad \text { for } \lambda>0,
$$

since $p_{m}$ is homogeneous of degree $m$.

Lemma A.1. - There exist $M>0$ and $R_{0}>1$ such that

$$
H_{p_{m}}^{2}\left(|x|^{2}\right) \geqslant M|\xi|^{2(m-1)}
$$

for any $(x, \xi) \in\left\{(y, \eta) \in T^{*} \mathbb{R}^{n}|| y\left|>R_{0},\right| \eta \mid \neq 0\right\}$.

Proof. - We have

$$
\begin{aligned}
& H_{p_{m}}^{2}\left(|x|^{2}\right)=2 H_{p_{m}}\left(x \cdot \partial_{\xi} p_{m}\right) \\
& \quad=2\left|\partial_{\xi} p_{m}\right|^{2}+2 \sum_{j, k=1}^{n} x_{j}\left(\partial_{x_{k}} \partial_{\xi_{j}} p_{m}\right) \partial_{\xi_{k}} p_{m}-2 \sum_{j, k=1}^{n} x_{j}\left(\partial_{\xi_{j}} \partial_{\xi_{k}} p_{m}\right) \partial_{x_{k}} p_{m} .
\end{aligned}
$$

On the other hand, by Assumption 1.1, there exists $C>0$ such that

$$
\left|2 \sum_{j, k=1}^{n} x_{j}\left(\partial_{x_{k}} \partial_{\xi_{j}} p_{m}\right) \partial_{\xi_{k}} p_{m}-2 \sum_{j, k=1}^{n} x_{j}\left(\partial_{\xi_{j}} \partial_{\xi_{k}} p_{m}\right) \partial_{x_{k}} p_{m}\right| \leqslant C\left\langle R_{0}\right\rangle^{-\mu}|\xi|^{2(m-1)} \text {. }
$$

Combining this with the non-degeneracy condition of $\partial_{\xi} p_{0}(\xi)$ in Assumption 1.1, we conclude the assertion.

Next, we observe that an energy bound on classical trajectories holds, even if $p$ is not elliptic. We note an analogous result is proved in [KPRV05], though our proof is simpler. 
Lemma A.2. - Fix $\left(x_{0}, \xi_{0}\right) \in T^{*} \mathbb{R}^{n}$ with $\xi_{0} \neq 0$ and suppose that $\left(x_{0}, \xi_{0}\right)$ is forward non-trapping in the sense that $\left|y\left(t, x_{0}, \xi_{0}\right)\right| \rightarrow \infty$ as $t \rightarrow \infty$. Then, there exist $C_{1}, C_{2}>0$ such that

$$
C_{1} \leqslant\left|\eta\left(t, x_{0}, \xi_{0}\right)\right| \leqslant C_{2}
$$

for $t \geqslant 0$.

Proof. - Let $R_{0}$ be as in Lemma A.1, and we let $R_{1} \geqslant R_{0}$ which is determined later. We first note that by the forward non-trapping condition and Lemma A.1, there exits $t_{0} \geqslant 0$ such that for $t \geqslant t_{0}$, we have

$$
\left|y\left(t, x_{0}, \xi_{0}\right)\right| \geqslant R_{1}, \quad \frac{d}{d t}\left|y\left(t, x_{0}, \xi_{0}\right)\right|^{2} \geqslant 0 .
$$

Indeed, it is easy to observe that there are $t_{0}>s_{0}>0$ such that $\frac{d^{2}}{d t^{2}}\left|y\left(t, x_{0}, \xi_{0}\right)\right|^{2}>0$ for $t \geqslant s_{0}$, and $\frac{d}{d t}\left|y\left(t_{0}, x_{0}, \xi_{0}\right)\right|^{2}>0$. Then for all $t \geqslant t_{0}$, the condition (A.1) is satisfied.

Let $C_{0}>0$ be a constant such that

$$
\left|\partial_{x} p_{m}(x, \xi)\right| \leqslant C_{0}|x|^{-1-\mu}|\xi|^{m}
$$

and we write $\eta_{0}=\left|\eta\left(t_{0}, x_{0}, \xi_{0}\right)\right|>0$. We set

$$
T=\sup \left\{s \geqslant t_{0}\left|\eta_{0} / 2 \leqslant\right| \eta\left(t, x_{0}, \xi_{0}\right) \mid \text { for } t \in\left[t_{0}, s\right]\right\} \in\left(t_{0}, \infty\right] .
$$

By Lemma A.1, we have

$$
\left|y\left(t, x_{0}, \xi_{0}\right)\right|^{2} \geqslant R_{1}^{2}+\frac{M \eta_{0}^{2(m-1)}}{2^{2 m-1}}\left(t-t_{0}\right)^{2}, \quad t_{0} \leqslant t \leqslant T .
$$

Now we note

$$
\left|\frac{d}{d t}\right| \eta\left(t, x_{0}, \xi_{0}\right)|| \leqslant C_{0}\left|y\left(t, x_{0}, \xi_{0}\right)\right|^{-1-\mu}\left|\eta\left(t, x_{0}, \xi_{0}\right)\right|^{m}
$$

and hence

$$
\left.\left|\frac{d}{d t}\right| \eta\left(t, x_{0}, \xi_{0}\right)\right|^{-(m-1)} \mid \leqslant(m-1) C_{0}\left(R_{1}^{2}+\frac{M \eta_{0}^{2(m-1)}}{2^{2 m-1}}\left(t-t_{0}\right)^{2}\right)^{-(1+\mu) / 2}
$$

for $t_{0} \leqslant t \leqslant T$. Thus we have

$$
\begin{aligned}
\left.\left|\eta_{0}^{-(m-1)}-\right| \eta\left(T, x_{0}, \xi_{0}\right)\right|^{-(m-1)} \mid & \\
& \leqslant \int_{t_{0}}^{T}(m-1) C_{0}\left(R_{1}^{2}+\frac{M \eta_{0}^{2(m-1)}}{2^{2 m-1}}\left(t-t_{0}\right)^{2}\right)^{-(1+\mu) / 2} d t \\
& \leqslant \frac{C_{\mu} C_{0} 2^{(2 m-1) / 2} R_{1}^{-\mu}}{\sqrt{M}} \eta_{0}^{-(m-1)},
\end{aligned}
$$

where $C_{\mu}=\int_{0}^{\infty}\left(1+s^{2}\right)^{-\frac{1+\mu}{2}} d s$. We now choose $R_{1}>0$ so large that

$$
\frac{C_{\mu} C_{0} 2^{(2 m-1) / 2} R_{1}^{-\mu}}{(1+\mu) \sqrt{M}}<1 / 2, \quad \text { i.e., } \quad R_{1}>\left(\frac{C_{\mu} C_{0} 2^{(2 m+1) / 2}}{(1+\mu) \sqrt{M}}\right)^{1 / \mu},
$$


then

$$
\left|\eta\left(T, x_{0}, \xi_{0}\right)\right|^{-(m-1)}<(3 / 2) \eta_{0}^{-(m-1)}
$$

i.e., $\left|\eta\left(T, x_{0}, \xi_{0}\right)\right|>(2 / 3)^{1 /(m-1)} \eta_{0}>(1 / 2) \eta_{0}$. If $T<\infty$, this is a contradiction, and hence $T=\infty$. Thus we also learn

$$
2^{-1} \eta_{0} \leqslant\left|\eta\left(t, x_{0}, \xi_{0}\right)\right| \leqslant 2^{1 /(m-1)} \eta_{0}, \quad t \geqslant t_{0}
$$

Corollary A.3. - Suppose the same assumptions as in Lemma A.2 hold. Moreover, suppose $\left|\xi_{0}\right|=1$. Then, we have

$$
C_{1} \lambda \leqslant\left|\eta\left(t, x_{0}, \lambda \xi_{0}\right)\right| \leqslant C_{2} \lambda
$$

for any $\lambda>0$ and $t \geqslant 0$.

Corollary A.4. - Under the same assumptions as in Lemma A.2 with $\left|\xi_{0}\right|=1$, there exist $C, C^{\prime}, K, K^{\prime}>0$ such that

$$
C \lambda t-K \leqslant\left|y\left(t, x_{0}, \lambda \xi_{0}\right)\right| \leqslant C^{\prime} \lambda t+K^{\prime}
$$

for $\lambda>0$ and $t \geqslant 0$.

Combining with the estimate $\left|\partial_{x} p_{m}(x, \xi)\right| \leqslant C\langle x\rangle^{-1-\mu}|\xi|^{m}$, we obtain:

Corollary A.5. - Suppose that $\left(x_{0}, \xi_{0}\right) \in \mathbb{R}^{n} \times \mathbb{R}^{n} \backslash\{0\}$ is non-trapping. Then,

$$
\begin{aligned}
& \eta_{+}=\lim _{t \rightarrow \infty} \eta\left(t, x_{0}, \xi_{0}\right) \neq 0, \\
& v_{+}=\lim _{t \rightarrow \infty} \partial_{\xi} p_{m}\left(y\left(t, x_{0}, \xi_{0}\right), \eta\left(t, x_{0}, \xi_{0}\right)\right)=\partial_{\xi} p_{0}\left(\eta_{+}\right) \neq 0
\end{aligned}
$$

exist.

\section{Appendix B. Construction of the conjugate operator}

Let $\left(x_{0}, \xi_{0}\right) \in p_{m}^{-1}(0) \backslash\{\xi=0\}$. By Assumption 1.2, $\left(x_{0}, \xi_{0}\right)$ is forward non-trapping. We denote $y(t)=y\left(t, x_{0}, \xi_{0}\right), \eta(t)=\eta\left(t, x_{0}, \xi_{0}\right)$. We note that

$$
\lim _{j \rightarrow \infty} \eta\left(t, x_{0}, \xi_{0}\right)=\eta_{+} \neq 0, \lim _{t \rightarrow \infty} \partial_{\xi} p_{m}(y(t), \eta(t))=v_{+} \neq 0,
$$

exist by Corollary A.5. Moreover, there exist $M_{1}, M_{2}>0$ such that

$$
\begin{aligned}
&\left|y(t) / t-v_{+}\right|,\left|\eta(t)-\eta_{+}\right|=O\left(\langle t\rangle^{-\mu}\right) \quad \text { as } t \rightarrow \infty, \\
& M_{1} \leqslant|\eta(t)| \leqslant M_{2}, \quad t \geqslant 0 .
\end{aligned}
$$

We denote $B(r, s, z, \zeta)=\left\{(x, \xi) \in \mathbb{R}^{2 n}|| z-x|<r,| \zeta-\xi \mid<s\right\} \subset \mathbb{R}^{2 n}$. In order to prove Proposition 3.3, it suffices to prove the following theorem. We set an $h$ dependent metric $g_{h}$ by

$$
g_{h}=d x^{2} /\langle x\rangle^{2}+h^{2 /(m-1)} d \xi^{2} .
$$

Theorem B.1. - There exist $\psi_{h} \in C_{c}^{\infty}\left(\mathbb{R}^{2 n}\right)$ and $\varphi_{h, t} \in C^{\infty}\left(\mathbb{R}_{\geqslant 0}, C_{c}^{\infty}\left(\mathbb{R}^{2 n}\right)\right)$ such that $F(h, t)=\operatorname{Op}\left(\varphi_{h, t}\right)$ and:

(i) $F(h, 0)=\left|\operatorname{Op}\left(\psi_{h}\right)\right|^{2}$ with $\psi_{h}\left(x_{0}, \xi_{0}\right) \geqslant 1$. 
(ii) $\varphi_{h, t}$ satisfies

$$
\operatorname{supp} \varphi_{h, t} \subset B\left(4 h^{-1} t \delta_{1}, 4 h^{-1 /(m-1)} \delta_{2}, h^{-1} t v_{+}, h^{-\frac{1}{m-1}} \eta_{+}\right)
$$

modulo $S\left(h^{\infty}, g_{h}\right)$ if $t / h$ is sufficiently large.

(iii) For any $\alpha, \beta \in \mathbb{N}_{\geqslant 0}^{n}$, there exists $C_{\alpha \beta}>0$ such that

$$
\left|\partial_{x}^{\alpha} \partial_{\xi}^{\beta} \varphi_{h, t}(x, \xi)\right| \leqslant C_{\alpha \beta}\langle t\rangle h^{(|\beta|+1) /(m-1)-1}\langle x\rangle^{-|\alpha|} .
$$

(iv) There exists a family of bounded operator $R(h, t)$ in $L^{2}\left(\mathbb{R}^{n}\right)$ such that

$$
\frac{\partial F}{\partial t}+i[P, F] \geqslant-R(h, t)
$$

where $\sup _{\geqslant 0}\langle t\rangle^{-1}\|R(h, t)\|_{L^{2} \rightarrow L^{2}}=O\left(h^{\infty}\right)$.

The proof of Theorem B.1 is based on the fact that any classical trajectory of $H_{p}$ behave as straight lines even if $p$ is not elliptic. We follow the argument in [Nak05].

Lemma B.2. - There exist constants $\delta_{1}, \delta_{2}>0$ with $\left|v_{+}\right|>4 \delta_{1}$ such that the following holds:

There exists a smooth function $\psi \in C^{\infty}\left(\mathbb{R}_{\geqslant 0}, C_{c}^{\infty}\left(\mathbb{R}^{2 n}\right)\right)$ such that

(i) $\psi \geqslant 0$, and $\psi\left(0, x_{0}, \xi_{0}\right) \geqslant 1$.

(ii) supp $\psi(t, \cdot, \cdot) \subset B\left(2 t \delta_{1}, 2 \delta_{2}, t v_{+}, \eta_{+}\right)$for $t \geqslant T_{0}$, where $T_{0}>0$ depends only on $\left(x_{0}, \xi_{0}\right), p_{m}$ and $\delta_{1}$.

(iii) For any $\alpha, \beta \in \mathbb{N}^{n}$, there exists $C_{\alpha \beta}>0$ such that

$$
\left|\partial_{x}^{\alpha} \partial_{\xi}^{\beta} \psi(t, x, \xi)\right| \leqslant C_{\alpha \beta}\langle x\rangle^{-|\alpha|},\left|\partial_{x}^{\alpha} \partial_{\xi}^{\beta} \partial_{t} \psi(t, x, \xi)\right| \leqslant C_{\alpha \beta}\langle x\rangle^{-1-|\alpha|}
$$

for $t \geqslant 0$ and $x, \xi \in \mathbb{R}^{n}$.

(iv) $\psi$ satisfies

$$
\left(\frac{\partial \psi}{\partial t}+\left\{p_{m}, \psi\right\}\right)(t, x, \xi) \geqslant 0
$$

for $t \geqslant 0, x, \xi \in \mathbb{R}^{n}$.

Proof. - Let $\Psi \in C^{\infty}(\mathbb{R})$ such that $0 \leqslant \Psi \leqslant 1, \Psi^{\prime} \leqslant 0, \Psi=1$ for $r \leqslant \frac{1}{2}, \Psi=0$ for $r \geqslant 1, \Psi(r)>0$ if $\frac{1}{2}<r<1$. We define

$$
\psi_{0}(t, x, \xi):=\Psi\left(\frac{|x-y(t)|}{\delta_{1}\langle t\rangle}\right) \Psi\left(\frac{|\xi-\eta(t)|}{\gamma(t)}\right)
$$

where we set $\gamma(t)=\delta_{2}-C_{1}\langle t\rangle^{-\mu}$ and let $C_{1}>0$ be determined later. We set

$$
\begin{aligned}
L(t, x, \xi) & =\partial_{\xi} p_{m}(x, \xi)-\partial_{\xi} p_{m}(y(t), \eta(t)), \\
A_{0}(t, x, \xi) & =\frac{1}{\delta_{1}\langle t\rangle}\left(L(t, x, \xi) \cdot \frac{x-y(t)}{|x-y(t)|}-\frac{t|x-y(t)|}{\langle t\rangle^{2}}\right), \\
A_{1}(t, x, \xi) & =\frac{1}{\gamma(t)}\left(-\frac{\gamma^{\prime}(t)|\xi-\eta(t)|}{\gamma(t)}+\left(\partial_{x} p_{m}(y(t), \eta(t))-\partial_{x} p_{m}(x, \xi)\right) \cdot \frac{\xi-\eta(t)}{|\xi-\eta(t)|}\right) .
\end{aligned}
$$


For $t>0$, we have

$$
\begin{aligned}
& \text { (B.2) } \quad\left(\frac{\partial \psi_{0}}{\partial t}+\left\{p_{m}, \psi_{0}\right\}\right)(t, x, \xi)= \\
& A_{0}(t, x, \xi) \Psi^{\prime}\left(\frac{|x-y(t)|}{\delta_{1} t}\right) \Psi\left(\frac{|\xi-\eta(t)|}{\gamma(t)}\right)+A_{1}(t, x, \xi) \Psi\left(\frac{|x-y(t)|}{\delta_{1} t}\right) \Psi^{\prime}\left(\frac{|\xi-\eta(t)|}{\gamma(t)}\right) .
\end{aligned}
$$

By Assumption 1.1 and (B.1), we have

$$
\left|\partial_{\xi} p_{m}(x, \xi)-\partial_{\xi} p_{m}(y(t), \eta(t))\right| \leqslant C_{0}|\xi-\eta(t)|+\frac{\delta_{1}}{4}
$$

for $(x, \xi) \in \operatorname{supp} \psi_{0}(t, \cdot), t \geqslant T_{0-1}$ with a constant $C_{0}>0$ and a time $T_{0-1}>0$. This implies

(B.3) $\delta_{1}\langle t\rangle A_{0}(t, x, \xi) \leqslant-\frac{\delta_{1} t}{2\langle t\rangle}+C_{0} \gamma(t)+\frac{\delta_{1}}{4} \leqslant-\frac{\delta_{1} t}{2\langle t\rangle}+C_{0} \delta_{2}-C_{0} C_{1}\langle t\rangle^{-\mu}+\frac{\delta_{1}}{4}$

for $(x, \xi) \in \operatorname{supp} \Psi^{\prime}\left(|x-y(t)| / \delta_{1}\langle t\rangle\right) \Psi(|\xi-\eta(t)| / \gamma(t))$. By Assumption 1.1 and (B.1), there exist $C>0, T_{00} \geqslant T_{0-1}$ such that for $(x, \xi) \in \operatorname{supp} \psi_{0}(t, x, \xi)$, we have

$$
\left|\partial_{x} p_{m}(y(t), \eta(t))-\partial_{x} p_{m}(x, \xi)\right| \leqslant C\langle t\rangle^{-1-\mu}
$$

for $t \geqslant T_{00}$. Here, we can choose $C>0$ independently of $C_{1}$. We note that $\gamma(t) / 2$ $\leqslant|\xi-\eta(t)|$ holds on the support of $\Psi^{\prime}(|\xi-\eta(t)| / \gamma(t))$. Using these observations, we learn

$$
\begin{aligned}
& A_{1}(t, x, \xi) \leqslant-\frac{\gamma^{\prime}(t)}{\gamma(t)^{2}}|\xi-\eta(t)|+\frac{C\langle t\rangle^{-1-\mu}}{\gamma(t)} \\
& \quad=\frac{1}{\gamma(t)}\left(-\frac{C_{1} \mu t}{\langle t\rangle^{2+\mu}} \cdot \frac{|\xi-\eta(t)|}{\gamma(t)}+\frac{C}{\langle t\rangle^{1+\mu}}\right) \leqslant-\frac{1}{\gamma(t)}\left(\frac{C_{1} \mu t}{2\langle t\rangle^{2+\mu}}-\frac{C}{\langle t\rangle^{1+\mu}}\right)
\end{aligned}
$$

for $(x, \xi) \in \operatorname{supp} \Psi\left(|x-y(t)| / \delta_{1}\langle t\rangle\right) \Psi^{\prime}(|\xi-\eta(t)| / \gamma(t))$ with $t \geqslant T_{00}$. By (B.2), (B.3) and (B.4) with $\Psi^{\prime} \leqslant 0$ and $\delta_{1}>>\delta_{2}$, we can select $T_{00}>0$ and $C_{1}>0$ such that for $t \geqslant T_{00}$,

$$
\left(\frac{\partial \psi_{0}}{\partial t}+\left\{p_{m}, \psi_{0}\right\}\right)(t, x, \xi) \geqslant 0
$$

Now we define $\psi(t, x, \xi)$ by the solution to (B.6)

$$
\begin{aligned}
\left(\frac{\partial \psi}{\partial t}+\left\{p_{m}, \psi\right\}\right)(t, x, \xi) & =\rho(t)\left(\frac{\partial \psi_{0}}{\partial t}+\left\{p_{m}, \psi_{0}\right\}\right)(t, x, \xi), \quad 0 \leqslant t \leqslant T_{00}+1 \\
\psi\left(T_{00}+1, x, \xi\right) & =\psi_{0}\left(T_{00}+1, x, \xi\right)
\end{aligned}
$$

where $\rho \in C^{\infty}(\mathbb{R},[0,1])$ such that $\rho(t)=1$ for $t \geqslant T_{00}+1, \rho(t)=0$ for $t \leqslant T_{00}$. Then we can extend $\psi$ smoothly to $t \geqslant T_{00}+1$ by $\psi(t, x, \xi)=\psi_{0}(t, x, \xi)$ for $t \geqslant T_{00}+1$. For $(x, \xi) \in \mathbb{R}^{2 n}$, by using $\rho(t) \leqslant 1$, we obtain

$$
\frac{d \psi}{d t}(t, y(t, x, \xi), \eta(t, x, \xi)) \leqslant \frac{d \psi_{0}}{d t}(t, y(t, x, \xi), \eta(t, x, \xi)) .
$$


Let $0 \leqslant s \leqslant T_{00}+1$. Integrating this inequality over $\left[s, T_{00}+1\right]$ with $(x, \xi)=\left(x_{0}, \xi_{0}\right)$ and using $\psi(t, x, \xi)=\psi_{0}(t, x, \xi)$ with $(t, x, \xi)=\left(T_{00}+1, y\left(T_{00}+1\right), \eta\left(T_{00}+1\right)\right)$, we have

$$
\psi(s, y(s), \eta(s)) \geqslant \psi_{0}(s, y(s), \eta(s)) \geqslant 0 .
$$

Substituting this inequality with $s=0$, we have $\psi\left(0, x_{0}, \xi_{0}\right) \geqslant \psi_{0}\left(0, x_{0}, \xi_{0}\right)=1$. This implies that $\psi$ satisfies (i). We set $T_{0}=T_{00}+1$. Now (ii) follows from (B.1) and the relation $\psi(t, x, \xi)=\psi_{0}(t, x, \xi)$ for $t \geqslant T_{0}$. (iv) follows from (B.5) and (B.6). Furthermore, (iii) follows from (B.1), (B.6), the relation $\psi(t, x, \xi)=\psi_{0}(t, x, \xi)$ for $t \geqslant T_{0}$ and the definition of $\psi_{0}$.

We set

$$
\psi_{h, t}(x, \xi)=\psi\left(t / h, x, h^{\frac{1}{m-1}} \xi\right), \quad \varphi_{0, h, t}(x, \xi)=\psi_{h, t} \# \psi_{h, t}(x, \xi),
$$

and $F_{0}(h, t)=\operatorname{Op}\left(\varphi_{0}(h, t, \cdot, \cdot)\right)=\left|\operatorname{Op}\left(\psi_{h, t}\right)\right|^{2}$, where \# denotes the composition of the Weyl quantization ([Zwo12, (4.3.6)] with $h=1$ ) and $|A|^{2}=A^{*} A$ for an operator $A$.

LEMMA B.3. -

(i) $F_{0}(0)=\left|\mathrm{Op}\left(\psi_{h, 0}\right)\right|^{2}$ with $\psi_{h, 0}\left(x_{0}, h^{-\frac{1}{m-1}} \xi_{0}\right) \geqslant 1$.

(ii) We have

$$
\operatorname{supp} \varphi_{0, h, t} \subset B\left(2 h^{-1} t \delta_{1}, 2 h^{-\frac{1}{m-1}} \delta_{2}, h^{-1} t v_{+}, h^{-\frac{1}{m-1}} \eta_{+}\right)
$$

modulo $S\left(h^{\infty}, g_{h}\right)$ if $t / h \geqslant T_{1}$.

(iii) For any $\alpha, \beta \in \mathbb{N}_{\geqslant 0}^{n}$, there exists $C_{\alpha \beta}>0$ such that

$$
\begin{aligned}
& \left|\partial_{x}^{\alpha} \partial_{\xi}^{\beta} \varphi_{0, h, t}(x, \xi)\right| \leqslant C_{\alpha \beta} h^{\frac{|\beta|}{m-1}}\langle x\rangle^{-|\alpha|}, \\
& \left|\partial_{x}^{\alpha} \partial_{\xi}^{\beta} \partial_{t} \varphi_{0, h, t}(x, \xi)\right| \leqslant C_{\alpha \beta} h^{\frac{|\beta|}{m-1}-1}\langle x\rangle^{-|\alpha|-1} .
\end{aligned}
$$

(iv) There exists $r_{0}(t, x, \xi) \in C^{\infty}\left(\mathbb{R}_{\geqslant 0} \times \mathbb{R}^{2 n}\right)$ such that

$$
\frac{\partial}{\partial t} F_{0}(h, t)+i\left[P, F_{0}(h, t)\right] \geqslant-\mathrm{Op}\left(r_{0, h, t}\right),
$$

and supp $r_{0, h, t} \subset \operatorname{supp} \varphi_{0, h, t}$ modulo $S\left(h^{\infty}\langle x\rangle^{-\infty}, g_{h}\right)$. Moreover, for any $\alpha, \beta \in \mathbb{N}_{\geqslant 0}^{n}$, there exists $C_{\alpha \beta}>0$ such that

$$
\left|\partial_{x}^{\alpha} \partial_{\xi}^{\beta} r_{0, h, t}(x, \xi)\right| \leqslant C_{\alpha \beta} h^{\frac{|\beta|-(m-2)}{m-1}}\langle x\rangle^{-|\alpha|-1-\mu} .
$$

Proof. - Properties (i)-(iii) follow from (B.1) and Lemma B.2. We prove (iv). Since $|x| \sim t / h$ holds on supp $\psi_{h, t}$, we learn $\partial_{t} \varphi_{0, h, t}(\cdot, \cdot) \in S\left(h^{-1}\langle x\rangle^{-1}, g_{h}\right)$. Moreover, we have $\left[P, F_{0}(h, t)\right] \in$ Op $S\left(\langle x\rangle^{-1}\langle\xi\rangle^{m-1}, g_{h}\right)$. By its support property, $\left[P, F_{0}\right.$ $(h, t)] \in \operatorname{Op} S\left(h^{-1}\langle x\rangle^{-1}, g_{h}\right)$ follows. We obtain

$$
\frac{\partial}{\partial t}\left|\psi_{h, t}(h, t, x, \xi)\right|^{2}+\left\{p_{m},\left|\psi_{h, t}(\cdot, \cdot)\right|^{2}\right\}(x, \xi) \geqslant 0
$$

by Lemma B.2(iv). We note $p=p_{m}+V$ with $V \in S^{m-1,-\mu}$ and

$$
\left[V, F_{0}(h, t)\right] \in \operatorname{Op} S\left(h^{-\frac{m-2}{m-1}}\langle x\rangle^{-1-\mu}, g_{h}\right) \text {. }
$$


By the sharp Gårding inequality, there exists $r_{0, h, t} \in S\left(h^{\frac{-(m-2)}{m-1}}\langle x\rangle^{-1-\mu}, g_{h}\right)$ such that (iv) holds.

Proof of Theorem B.1. - We choose $\lambda_{0}, \lambda_{1}, \lambda_{2}, \cdots \in[1,2)$ such that

$$
1=\lambda_{0}<\lambda_{1}<\lambda_{2}<\cdots<2,
$$

and take $\psi_{k, h, t}(x, \xi)$ as $\psi_{h, t}(x, \xi)$ and $T_{k}$ as $T_{0}$ with $\delta_{j}$ replaced by $\lambda_{k} \delta_{j}$ in Lemma B.2 and (B.7). By the choice of $\Psi$, we note

$$
\psi_{k+1, h, t}(x, \xi) \geqslant L_{k}
$$

on $\operatorname{supp} \psi_{k, h, t}(\cdot, \cdot)$ for some $L_{k}>0$. For $k \geqslant 1$, set

$$
\varphi_{k, h, t}(x, \xi)=h^{\frac{k-m+1}{m-1}} t C_{k} \psi_{k, h, t} \# \psi_{k, h, t} \in S\left(h^{\frac{k-m+1}{m-1}} t, g_{h}\right)
$$

where $C_{k}>0$ is determined later. By Lemma B.3(iv), we can write $r_{0, h, t}=r_{01, h, t}+$ $r_{02, h, t}$, where

$$
r_{01, h, t} \in S\left(h^{\frac{-(m-2)}{m-1}}\langle x\rangle^{-1-\mu}, g_{h}\right)
$$

satisfies supp $r_{01, h, t}(t, \cdot, \cdot) \subset \operatorname{supp} \varphi_{0}(t, \cdot, \cdot)$ and $r_{02, h, t} \in S\left(h^{\infty}\langle x\rangle^{-\infty}, g_{h}\right)$. By (B.8), we can find $C_{1}>0$ such that

$$
r_{01, h, t}(x, \xi) \leqslant C_{1} h^{\frac{-m+2}{m-1}}\left|\psi_{1, h, t}(x, \xi)\right|^{2} .
$$

This inequality with Lemma B.2(iv) implies

$$
\begin{aligned}
& C_{1} h^{\frac{-m+2}{m-1}}\left(\frac{\partial}{\partial t}\left(t\left|\psi_{1, h, t}\right|^{2}\right)+t\left\{p_{m},\left|\psi_{1, h, t}\right|^{2}\right\}\right)(x, \xi) \\
& =C_{1} h^{\frac{-m+2}{m-1}} t\left(\frac{\partial}{\partial t}\left|\psi_{1, h, t}\right|^{2}+\left\{p_{m},\left|\psi_{1, h, t}\right|^{2}\right\}\right)(x, \xi)+C_{1} h^{\frac{-m+2}{m-1}}\left|\psi_{1, h, t}(x, \xi)\right|^{2} \\
& \geqslant r_{01, h, t}(x, \xi) .
\end{aligned}
$$

Taking $M_{k}=\max \left(T_{k},|| v_{+}\left|-2 \lambda_{k} \delta_{1}\right|^{-1}\right)>0$, we have

$$
t \leqslant M_{k} h\langle x\rangle, \text { for }(t, x, \xi) \in \operatorname{supp} \psi_{k, h, t}
$$

by Lemma B.3(ii). Lemma B.3(iii) with (B.11) implies

$$
\left.h^{\frac{-m+2}{m-1}} t\left(\frac{\partial\left|\psi_{1, h, t}\right|^{2}}{\partial t}+\left\{p_{m},\left|\psi_{1, h, t}\right|^{2}\right\}\right) \in S\left(h^{\frac{-m+2}{m-1}}, g_{h}\right)\right) \text {. }
$$

By (B.9), (B.10) and (B.12), it follows that the both sides in (B.10) belong to $S\left(h^{\frac{-m+2}{m-1}}, d x^{2} /\langle x\rangle^{2}+h^{2 /(m-1)} d \xi^{2}\right)$. The sharp Gårding inequality implies that there exists

$$
r_{1, h, t} \in S\left(h^{\frac{-m+3}{m-1}}\langle x\rangle^{-1}, g_{h}\right)
$$

which is supported in supp $\varphi_{1, h, t}$ modulo $S\left(h^{\infty}\langle x\rangle^{-\infty}, g_{h}\right)$ such that

$$
\frac{\partial}{\partial t} \operatorname{Op}\left(\varphi_{1, h, t}\right)+i\left[P, \operatorname{Op}\left(\varphi_{1, h, t}\right)\right] \geqslant \operatorname{Op}\left(r_{0, h, t}\right)-\operatorname{Op}\left(r_{1, h, t}\right) .
$$

We set $F_{1}(h, t)=F_{0}(h, t)+\operatorname{Op}\left(\varphi_{1, h, t}\right)$, then we have

$$
\frac{\partial}{\partial t} F_{1}(h, t)+i\left[P, F_{1}(h, t)\right] \geqslant-\mathrm{Op}\left(r_{1, t, h}\right) .
$$


Iterating the above argument, we can construct $C_{k}>0, F_{k}(t)$ and

$$
r_{k, h, t} \in S\left(h^{\frac{k-m+2}{m-1}}\langle x\rangle^{-1}, g_{h}\right)
$$

such that supp $r_{k, h, t} \subset \operatorname{supp} \varphi_{k, h, t}(\cdot, \cdot)$ modulo $S\left(h^{\infty}\langle x\rangle^{-\infty}, g_{h}\right)$ and

$$
\begin{aligned}
& \frac{\partial}{\partial t} F_{k}(h, t)+i\left[P, F_{k}(h, t)\right] \geqslant-\mathrm{Op}\left(r_{k, h, t}(\cdot, \cdot)\right), \\
& F_{k+1}(h, t)=F_{k}(h, t)+\operatorname{Op}\left(\varphi_{k, h, t}\right), \\
& r_{k, h, t}(x, \xi) \leqslant C_{k+1} h^{\frac{k-m+2}{m-1}}\left|\psi_{k+1, h, t}(x, \xi)\right|^{2} \text { modulo } S\left(h^{\infty}\langle x\rangle^{-\infty}, g_{h}\right) .
\end{aligned}
$$

By the Borel's Theorem (see [Theorem 4.15][Zwo12]), we can define

$$
\varphi_{h, t}(x, \xi) \sim \sum_{k=0}^{\infty} \varphi_{k, h, t}(x, \xi)
$$

and $F(h, t)=\mathrm{Op}\left(\varphi_{h, t}\right)$. Then, $F(h, t)$ satisfies the properties in Theorem B.1. This completes the proof of Theorem B.1.

\section{Appendix C. Compactly supported perturbation}

The proof is considerably simpler if the perturbation is compactly supported, since we do not need the argument of Subsection 3.2. Here we discuss the simpler argument for this case. We assume that there exists $R>0$ such that $\operatorname{supp} q \subset B_{R}(0) \times \mathbb{R}^{n}$, where $B_{R}(0)=\left\{x \in \mathbb{R}^{n}|| x \mid<R\right\}$. We note still the local regularity argument (Subsection 3.1 and Appendices A, B). Let $\psi \in C^{\infty}\left(\mathbb{R}^{n}\right)$ be a real-valued function such that $\psi=1$ on $\mathbb{R}^{n} \backslash B_{R+1}(0)$ and $\psi=0$ on $B_{R}(0)$.

Proposition C.1. - Let $k \geqslant 0$ and $u \in L^{2}\left(\mathbb{R}^{n}\right) \cap H_{\mathrm{loc}}^{k+m-1}\left(\mathbb{R}^{n}\right)$ be a distributional solution to $(P+i) u=0$. Then we have $\psi u \in H^{k}$. In particular, $u \in H^{k}$ follows.

Proof. - Set $N=I-\Delta$ and $N_{\varepsilon}=(I-\Delta)(I-\varepsilon \Delta)^{-1}$ and define $L=p_{0}(D)$ where $\Delta$ denotes the standard Laplacian on $\mathbb{R}^{n}$. By virtue of the support property of $\psi$, we compute

$$
L(\psi u)=P(\psi u)=\psi P u+[P, \psi] u=-i \psi u+K u,
$$

where $K:=[P, \psi]$ is compactly supported coefficients differential operator with order $m-1$. We note $K u \in H^{1}$ since $u \in H_{\text {loc }}^{m}\left(\mathbb{R}^{n}\right)$. Hence, we have

$$
\begin{aligned}
2 i \operatorname{Im}\left(N_{\varepsilon}^{2 k}(\psi u), L(\psi u)\right)_{L^{2}} & =2 i \operatorname{Im}\left(N_{\varepsilon}^{2 k}(\psi u),-i \psi u+K u\right)_{L^{2}} \\
& =-2 i\left\|N_{\varepsilon}^{k}(\psi u)\right\|_{L^{2}}^{2}+2 i \operatorname{Im}\left(N_{\varepsilon}^{2 k}(\psi u), K u\right)_{L^{2}} .
\end{aligned}
$$

On the other hand, by the Plancherel theorem, we have

$$
2 i \operatorname{Im}\left(N_{\varepsilon}^{2 k}(\psi u), L(\psi u)\right)_{L^{2}}=\left(N_{\varepsilon}^{2 k}(\psi u), L(\psi u)\right)_{L^{2}}-\left(L(\psi u), N_{\varepsilon}^{2 k}(\psi u)\right)_{L^{2}}=0 .
$$

Thus, we have

$$
\left\|N_{\varepsilon}^{k}(\psi u)\right\|_{L^{2}}^{2} \leqslant\left|\operatorname{Im}\left(N_{\varepsilon}^{2 k}(\psi u), K u\right)\right| \leqslant\left\|N_{\varepsilon}^{k}(\psi u)\right\|_{L^{2}}\left\|N_{\varepsilon}^{k} K u\right\|_{L^{2}}
$$


Consequently, take $\varepsilon \rightarrow 0$ and we obtain $\left\|N^{k}(\psi u)\right\|_{L^{2}} \leqslant\left\|N^{k} K u\right\|_{L^{2}}<\infty$, by using the monotone convergence theorem and $K u \in H^{k}$. This implies $\psi u \in H^{k}$.

Proof of Proposition 3.1. - Suppose that $u \in L^{2}\left(\mathbb{R}^{n}\right)$ satisfies $(P+i) u=0$. By Proposition 3.2, we have $u \in C^{\infty}\left(\mathbb{R}^{n}\right) \subset H_{\text {loc }}^{3(m-1) / 2}\left(\mathbb{R}^{n}\right)$. By Proposition C.1, we conclude $u \in H^{(m-1) / 2} \subset H^{(m-1) / 2,-1 / 2}$.

\section{BIBLIOGRAPHY}

[BVW15] Dean Baskin, András Vasy, and Jared Wunsch, Asymptotics of radiation fields in asymptotically Minkowski space, Am. J. Math. 137 (2015), no. 5, 1293-1364. $\uparrow 1037$

[Chi02] Hiroyuki Chihara, Smoothing effects of dispersive pseudodifferential equations, Commun. Partial Differ. Equations 27 (2002), no. 9-10, 1953-2005. $\uparrow 1037$

[DH72] Johannes J. Duistermaat and Lars Hörmander, Fourier integral operators. II, Acta Math. 128 (1972), no. 3-4, 183-269. 11037

[GRHV16] Jesse Gell-Redman, Nick Haber, and András Vasy, The Feynman propagator on perturbations of Minkowski space, Commun. Math. Phys. 342 (2016), no. 1, 333-384. $\uparrow 1037$

[GS93] Jean-Michel Ghidaglia and Jean-Claude Saut, Nonelliptic Schrödinger equations, J. Nonlinear Sci. 3 (1993), no. 2, 169-195. $\uparrow 1037$

[GT12] Nicolas Godet and Nikolay Tzvetkov, Strichartz estimates for the periodic non-elliptic Schrödinger equation, C. R. Math. Acad. Sci. Paris 350 (2012), no. 21-22, 955-958. $\uparrow 1037$

[GW19] Christian Gérard and Michal Wrochna, The massive Feynman propagator on asymptotically Minkowski spacetimes, Amer. J. Math. 19 (2019), no. 6, 1501-1546. $\uparrow 1037$

[Hör85] Lars Hörmander, Analysis of Linear Partial Differential Operators, Grundlehren der Mathematischen Wissenschaften, vol. I-IV, Springer, 1983-1985. $\uparrow 1037,1038$

[IN09] Kenichi Ito and Shu Nakamura, Singularities of solutions to Schrödinger equation on scattering manifold, Am. J. Math. 131 (2009), no. 6, 1835-1865. 11037

[KPRV05] Carlos E. Kenig, Gustavo Ponce, Christian Rolvung, and Luis Vega, Variable coefficient Schrödinger flows for ultrahyperbolic operators, Adv. Math. 196 (2005), no. 2, 373-486. $\uparrow 1038,1050$

[Mel94] Richard B. Melrose, Spectral and scattering theory for the Laplacian on asymptotically Euclidian spaces, Spectral and scattering theory. Proceedings of the Taniguchi international workshop, held at Sanda, Hyogo, Japan, Lecture Notes in Pure and Applied Mathematics, vol. 161, Basel: Marcel Dekker, 1994, pp. 85-130. $\uparrow 1038,1040$

[MT15] Haruya Mizutani and Nikolay Tzvetkov, Strichartz estimates for non-elliptic Schrödinger equations on compact manifolds, Commun. Partial Differ. Equations 40 (2015), no. 6, 1182-1195. $\uparrow 1037$

[Nak05] Shu Nakamura, Propagation of the homogeneous wave front set for Schrödinger equations, Duke Math. J. 126 (2005), no. 2, 349-367. ^1037, 1040, 1053

[RS80] Michael Reed and Barry Simon, The Methods of Modern Mathematical Physics, vol. I-IV, Academic Press Inc., 1972-1980. $\uparrow 1037,1039$

[Sal07] Delphine Salort, The Schrödinger equation type with a nonelliptic operator, Commun. Partial Differ. Equations 32 (2007), no. 1-3, 209-228. $\uparrow 1037$

[Tai20] Kouichi Taira, Strichartz estimates for non-degenerate Schrödinger equations, Math. Nachr. 293 (2020), no. 4, 774-793. $\uparrow 1037$

[Vas20] András Vasy, Essential self-adjointness of the wave operator and the limiting absorption principle on Lorentzian scattering spaces, J. Spectr. Theory 10 (2020), no. 2, 439-461. $\uparrow 1037,1039$ 
[VW18] András Vasy and Michal Wrochna, Quantum fields from global propagators on asymptotically Minkowski and extended de Sitter spacetimes, Ann. Henri Poincaré 19 (2018), no. $5,1529-1586$. $\uparrow 1037$

[Wan13] Yuzhao Wang, Periodic cubic hyperbolic Schrödinger equation on $\mathbb{T}^{2}$, J. Funct. Anal. 265 (2013), no. 3, 424-434. $\uparrow 1037$

[Zwo12] Maciej Zworski, Semiclassical Analysis, Graduate Studies in Mathematics, vol. 138, American Mathematical Society, 2012. $\uparrow 1055,1057$

Manuscript received on 30th January 2020,

revised on 12th November 2020,

accepted on 21st December 2020.

Recommended by Editor F. Hérau.

Published under license CC BY 4.0.

(c) (i)

This journal is a member of Centre Mersenne.

CENTR

MERSENNE

Shu NAKAMURA

Department of Mathematics,

Faculty of Sciences,

Gakushuin University,

1-5-1, Mejiro, Toshima,

Tokyo, (Japan) 171-8588

shu.nakamura@gakushuin.ac.jp

Kouichi TAIRA

Research Organization

of Science and Technology,

Ritsumeikan university,

Kita Ward, Kyoto, (Japan)

20v00029@gst.ritsumei.ac.jp 